\title{
ON THE ALGEBRAIC AND ARITHMETIC STRUCTURE OF THE MONOID OF PRODUCT-ONE SEQUENCES
}

\author{
JUN SEOK OH
}

\begin{abstract}
Let $G$ be a finite group. A finite unordered sequence $S=g_{1} \cdot \ldots \cdot g_{\ell}$ of terms from $G$, where repetition is allowed, is a product-one sequence if its terms can be ordered such that their product equals $1_{G}$, the identity element of the group. As usual, we consider sequences as elements of the free abelian monoid $\mathcal{F}(G)$ with basis $G$, and we study the submonoid $\mathcal{B}(G) \subset \mathcal{F}(G)$ of all product-one sequences. This is a finitely generated C-monoid, which is a Krull monoid if and only if $G$ is abelian. In case of abelian groups, $\mathcal{B}(G)$ is a well-studied object. In the present paper we focus on non-abelian groups, and we study the class semigroup and the arithmetic of $\mathcal{B}(G)$.
\end{abstract}

\section{INTRODUCTION}

Let $G$ be a finite group. By a sequence over $G$, we mean a finite unordered sequence of terms from $G$, where the repetition of elements is allowed (the terminology stems from Arithmetic Combinatorics). A sequence $S$ is a product-one sequence if its terms can be ordered such that their product equals the identity element of the group. Clearly, juxtaposition of sequences is a commutative operation on the set of sequences. As usual we consider sequences as elements of the free abelian monoid $\mathcal{F}(G)$ with basis $G$, and clearly the subset $\mathcal{B}(G) \subset \mathcal{F}(G)$ of all product-one sequences is a submonoid. The small Davenport constant $\mathrm{d}(G)$ is the maximal integer $\ell$ for which there is a sequence of length $\ell$ which has no productone subsequence. The large Davenport constant $\mathrm{D}(G)$ is the maximal length of a minimal product-one sequence (by a minimal product-one sequence we mean an irreducible element in the monoid $\mathcal{B}(G)$ ).

Suppose that $G$ is abelian, and let us use additive notation in this case. Then product-one sequences are zero-sum sequences and their study is the main objective of Zero-Sum Theory (12, 25). The monoid $\mathcal{B}(G)$ is a Krull monoid with class group $G$ (apart from the exceptional case where $|G|=2$ ), and because it has intimate relationship with general Krull monoids having class group $G$, the study of $\mathcal{B}(G)$ is a central topic in factorization theory ([18, 15]).

Although the abelian setting has been the dominant one, many of the combinatorial problems on sequences over abelian groups have also been studied in the nonabelian setting. For example, an upper bound on the small Davenport constant was given already in the 1970s [29], and in recent years Gao's Theorem $\mathrm{E}(G)=|G|+\mathrm{d}(G)$ ([25. Chapter 16]) has been generalized to a variety of nonabelian groups ([3, 14, 13, 26, 27]). Fresh impetus came from invariant theory. If $G$ is abelian, then $\mathrm{d}(G)+1=\boldsymbol{\beta}(G)=$ $\mathrm{D}(G)$, where $\boldsymbol{\beta}(G)$ is the Noether number. If $G$ is nonabelian and has a cyclic subgroup of index two, then the Davenport constants and the Noether number have been explicitely determined ( 8 , 17]), and it turned out that $\mathrm{d}(G)+1 \leq \boldsymbol{\beta}(G) \leq \mathrm{D}(G)$. For a survey on the interplay with invariant theory we refer to 9] and for recent progress to [24, 6, 7, 10].

Let $F$ be a factorial monoid. A submonoid $H \subset F$ is a C-monoid if $H^{\times}=H \cap F^{\times}$and the reduced class semigroup is finite. A commutative ring is called a C-ring if its monoid of regular elements is a C-monoid. To give an example of a C-ring, consider a Mori domain $R$. If its conductor $\mathfrak{f}=(R: \widehat{R})$ is

2010 Mathematics Subject Classification. 13A50, 20D60, $20 \mathrm{M} 13$.

Key words and phrases. product-one sequences, Davenport constant, C-monoids, sets of lengths.

This work was supported by the Austrian Science Fund FWF, W1230 Doctoral Program Discrete Mathematics. 
nonzero and the residue class $\operatorname{ring} \widehat{R} / \mathfrak{f}$ is finite, then $R$ is a C-ring. For more on C-rings we refer to [30, 21]. The finiteness of the reduced class semigroup is used to derive arithmetical finiteness result for C-monoids ([18]). However, the structure of the (reduced) class semigroup has never been studied before.

The monoid $\mathcal{B}(G)$ of product-one sequences is a finitely generated C-monoid, which is Krull if and only if $G$ is abelian (in the Krull case, the class semigroup coincides with the class group which is isomorphic to $G$, apart from the exceptional case where $|G|=2$ ). After putting together the needed background in Section 2, we study the structure of the class semigroup of $\mathcal{B}(G)$ in Section 3 . Among others we show that the unit group of the class semigroup is isomorphic to the center of $G$, and we reveal a subgroup of the class semigroup which is isomorphic to $G / G^{\prime}$, which is the class group of the complete integral closure of $\mathcal{B}(G)$ (see Theorems 3.8 and 3.10, $G^{\prime}$ denotes the commutator subgroup of $G$ ). In Section 4 we provide a complete description of the class semigroup for non-abelian groups of small order. In Section 5 we study the arithmetic of $\mathcal{B}(G)$ and our focus is on sets of lengths. Among others we show that unions of sets of lengths are finite intervals (Theorem 5.5 ).

Throughout this paper, let $G$ be a multiplicatively written finite group with identity element $1_{G} \in G$.

\section{Preliminaries}

We denote by $\mathbb{N}$ the set of positive integers. For integers $a, b \in \mathbb{Z},[a, b]=\{x \in \mathbb{Z} \mid a \leq x \leq b\}$ means the discrete interval. For every $n \in \mathbb{N}, C_{n}$ denotes a cyclic group of order $n$. For an element $g \in G$, $\operatorname{ord}(g) \in \mathbb{N}$ is its order, and for a subset $G_{0} \subset G,\left\langle G_{0}\right\rangle \subset G$ denotes the subgroup generated by $G_{0}$. We will use the following standard notation of group theory:

- $\mathrm{Z}(G)=\{g \in G \mid g x=x g$ for all $x \in G\} \triangleleft G$ is the center of $G$,

- $[x, y]=x y x^{-1} y^{-1} \in G$ is the commutator of the elements $x, y \in G$, and

- $G^{\prime}=[G, G]=\langle[x, y] \mid x, y \in G\rangle \triangleleft G$ is the commutator subgroup of $G$.

Semigroups. All our semigroups are commutative and have an identity element. Let $S$ be a semigroup. We denote by $S^{\times}$its group of invertible elements and by $\mathrm{E}(S)$ the set of all idempotents of $S$. $\mathrm{E}(S)$ is endowed with the Rees order $\leq$, defined by $e \leq f$ if $e f=e$. Clearly, $e f \leq e$ and $e f \leq f$ for all $e, f \in \mathrm{E}(S)$. If $E \subset \mathrm{E}(S)$ is a finite subsemigroup, then $E$ has a smallest element.

By a monoid, we mean a semigroup which satisfies the cancellation laws. Let $H$ be a monoid. Then $\mathrm{q}(H)$ denotes the quotient group of $H$ and $\mathcal{A}(H)$ the set of irreducibles (atoms) of $H$. The monoid $H$ is called atomic if every non-unit of $H$ can be written as a finite product of atoms. We say that $H$ is reduced if $H^{\times}=\{1\}$, and we denote by $H_{\text {red }}=H / H^{\times}=\left\{a H^{\times} \mid a \in H\right\}$ the associated reduced monoid of $H$. A monoid $F$ is called free abelian with basis $P \subset F$ if every $a \in F$ has a unique representation of the form

$$
a=\prod_{p \in P} p^{v_{p}(a)} \quad \text { with } \quad \vee_{p}(a)=0 \text { for almost all } p \in P .
$$

If $F$ is free abelian with basis $P$, then $P$ is the set of primes of $F$, we set $F=\mathcal{F}(P)$, and denote by

- $|a|=\sum_{p \in P} v_{p}(a)$ the length of $a$, and by

- $\operatorname{supp}(a)=\left\{p \in P \mid \mathrm{v}_{p}(a)>0\right\}$ the support of $a$.

A monoid $F$ is factorial if and only if $F_{\text {red }}$ is free abelian if and only if $F$ is atomic and every atom is a prime. We denote by

- $H^{\prime}=\left\{x \in \mathrm{q}(H) \mid\right.$ there is an $N \in \mathbb{N}$ such that $x^{n} \in H$ for all $\left.n \geq N\right\}$ the seminormalization of $H$, by

- $\widetilde{H}=\left\{x \in \mathrm{q}(H) \mid x^{N} \in H\right.$ for some $\left.N \in \mathbb{N}\right\}$ the root closure of $H$, and by

- $\widehat{H}=\left\{x \in \mathrm{q}(H) \mid\right.$ there is a $c \in H$ such that $c x^{n} \in H$ for all $\left.n \in \mathbb{N}\right\}$ the complete integral closure of $H$,

and observe that $H \subset H^{\prime} \subset \widetilde{H} \subset \widehat{H} \subset \mathrm{q}(H)$. Then the monoid $H$ is called 
- seminormal if $H=H^{\prime}$ (equivalently, if $x \in \mathrm{q}(H)$ and $x^{2}, x^{3} \in H$, then $x \in H$ ),

- root closed if $H=\widetilde{H}$,

- completely integrally closed if $H=\widehat{H}$.

If $D$ is a monoid and $H \subset D$ a submonoid, then $H$ is a divisor-closed submonoid if $a \in H, b \in D$, and $b \mid a$ implies that $b \in H$. A monoid homomorphism $\varphi: H \rightarrow D$ is said to be

- cofinal if for every $\alpha \in D$ there is an $a \in H$ such that $\alpha \mid \varphi(a)$.

- a divisor homomorphism if $a, b \in H$ and $\varphi(a) \mid \varphi(b)$ implies that $a \mid b$.

- a divisor theory if $D$ is free abelian, $\varphi$ is a divisor homomorphism, and for all $\alpha \in D$ there are $a_{1}, \ldots, a_{m} \in H$ such that $\alpha=\operatorname{gcd}\left(\varphi\left(a_{1}\right), \ldots, \varphi\left(a_{m}\right)\right)$.

- a transfer homomorphism if it satisfies the following two properties:

(T 1) $D=\varphi(H) D^{\times}$and $\varphi^{-1}\left(D^{\times}\right)=H^{\times}$.

(T 2) If $u \in H, b, c \in D$ and $\varphi(u)=b c$, then there exist $v, w \in H$ such that $u=v w$, $\varphi(v) D^{\times}=b D^{\times}$, and $\varphi(w) D^{\times}=c D^{\times}$.

Transfer Krull monoids. A monoid $H$ is said to be a Krull monoid if it satisfies one of the following equivalent conditions (see [18, Theorem 2.4.8]):

(a) $H$ is completely integrally closed and satisfies the ACC on divisorial ideals.

(b) There is a divisor homomorphism $\varphi: H \rightarrow F$, where $F$ is free abelian monoid.

(c) $H$ has a divisor theory.

A commutative domain $R$ is a Krull domain if and only if its multiplicative monoid of nonzero elements is Krull. Further examples of Krull monoids can be found in [18, 16. Let $H$ be a Krull monoid. Then a divisor theory $\varphi: H \rightarrow F$ is unique up to isomorphism and

$$
\mathcal{C}(H)=\mathrm{q}(F) / \mathrm{q}(\varphi(H))
$$

is called the class group of $H$.

A monoid $H$ is said to be a transfer Krull monoid if it allows a transfer homomorphism to a Krull monoid (since in our setting all monoids are commutative, 2, Lemma 2.3.3] shows that the present definition coincides with the definition in [16]). The significance of transfer homomorphisms $\theta: H \rightarrow B$ stems from the fact that they allow to pull back arithmetical properties from the (simpler monoid) $B$ to the monoid $H$ (of original interest). In particular, if $H$ is a transfer Krull monoid, then sets of lengths in $H$ coincide with sets of lengths in a Krull monoid (see Equation (5.1)).

Since the identity map is a transfer homomorphism, every Krull monoid is a transfer Krull monoid. For a list of transfer Krull monoids which are not Krull we refer to [16. To give one such example, consider a ring of integers $\mathcal{O}$ in an algebraic number field $K$, a central simple algebra $A$ over $K$, and a classical maximal $\mathcal{O}$-order $R$ of $A$. If every stably free left $R$-ideal is free, then the (non-commutative) semigroup of cancellative elements of $R$ is a transfer Krull monoid over a finite abelian group ([33]).

Class semigroups and C-monoids. (a detailed presentation can be found in [18, Chapter 2]). Let $F$ be a monoid and $H \subset F$ a submonoid. Two elements $y, y^{\prime} \in F$ are called $H$-equivalent, denote $y \sim_{H} y^{\prime}$, if $y^{-1} H \cap F=y^{\prime-1} H \cap F$. $H$-equivalence is a congruence relation on $F$. For $y \in F$, let $[y]_{H}^{F}$ denote the congruence class of $y$, and let

$$
\mathcal{C}(H, F)=\left\{[y]_{H}^{F} \mid y \in F\right\} \quad \text { and } \quad \mathcal{C}^{*}(H, F)=\left\{[y]_{H}^{F} \mid y \in\left(F \backslash F^{\times}\right) \cup\{1\}\right\} .
$$

Then $\mathcal{C}(H, F)$ is a commutative semigroup with unit element $[1]_{H}^{F}$ (called the class semigroup of $H$ in $F$ ) and $\mathcal{C}^{*}(H, F) \subset \mathcal{C}(H, F)$ is a subsemigroup (called the reduced class semigroup of $H$ in $F$ ).

As usual, class groups and class semigroups will both be written additively. The following lemma describes the relationship between class groups and class semigroups. Its proof is elementary and can be found in [18, Propositions 2.8.7 and 2.8.8]

Lemma 2.1. Let $F$ be a monoid and $H \subset F$ be a submonoid. 
1. If $H \subset F$ is cofinal, then the map $\theta: \mathcal{C}(H, F) \rightarrow \mathrm{q}(F) / \mathrm{q}(H),[y]_{H}^{F} \mapsto y \mathrm{q}(H)$ for all $y \in F$, is an epimorphism. Moreover, $\theta$ is an isomorphism if and only if $H \hookrightarrow F$ is a divisor homomorphism.

2. If $\mathcal{C}(H, F)$ is a group, then $H \subset F$ is cofinal, and if $\mathcal{C}(H, F)$ is a torsion group, then $H \hookrightarrow F$ is a divisor homomorphism.

A monoid $H$ is called a $\mathrm{C}$-monoid if $H$ is a submonoid of a factorial monoid $F$ such that $H \cap F^{\times}=H^{\times}$ and $\mathcal{C}^{*}(H, F)$ is finite. A commutative ring $R$ is a $\mathrm{C}$-ring if its monoid of regular elements is a C-monoid. A Krull monoid is a C-monoid if and only if it has finite class group. We refer to [18, 30, 21, 28] for more on C-monoids. To give an explicit example, consider a Mori ring $R$. If the conductor $\mathfrak{f}=(R: \widehat{R})$ is nonzero and $\widehat{R} / \mathfrak{f}$ is finite, then $R$ is a C-ring. We will need the following lemma (for a proof see [18, Theorem 2.9.11]).

Lemma 2.2. Let $F=F^{\times} \times \mathcal{F}(P)$ be factorial and $H \subset F$ be a C-monoid. Suppose that $\mathrm{v}_{p}(H) \subset \mathbb{N}_{0}$ is a numerical monoid for all $p \in P$ (this is a minimality condition on $F$ which can always be assumed without restriction).

1. $\widehat{H}$ is a Krull monoid with nonempty conductor $(H: \widehat{H})$ and finite class group $\mathcal{C}(\widehat{H})$.

2. The map $\partial: \widehat{H} \rightarrow \mathcal{F}(P)$, defined by

$$
\partial(a)=\prod_{p \in P} p^{\mathrm{v}_{p}(a)}
$$

is a divisor theory, and there is an epimorphism $\mathcal{C}^{*}(H, F) \rightarrow \mathcal{C}(\widehat{H})$.

\section{Algebraic Properties of the monoid of Product-one Sequences}

We introduce sequences over the finite group $G$. Our notation and terminology follows the articles [17, 24, 9]. Let $G_{0} \subset G$ be a subset. The elements of the free abelian monoid $\mathcal{F}\left(G_{0}\right)$ will be called sequences over $G_{0}$. This terminology goes back to Arithmetic Combinatorics. Indeed, a sequence over $G_{0}$ can be viewed as a finite unordered sequence of terms from $G_{0}$, where the repetition of elements is allowed. In order to avoid confusion between the multiplication in $G$ and multiplication in $\mathcal{F}\left(G_{0}\right)$, we denote multiplication in $\mathcal{F}\left(G_{0}\right)$ by the boldsymbol $\cdot$ and we use brackets for all exponentiation in $\mathcal{F}\left(G_{0}\right)$. In particular, a sequence $S \in \mathcal{F}\left(G_{0}\right)$ has the form

$$
S=g_{1} \cdot \ldots \cdot g_{\ell}=\underset{i \in[1, \ell]}{\bullet} g_{i}=\underset{g \in G_{0}}{\bullet} g^{\left[v_{g}(S)\right]} \in \mathcal{F}\left(G_{0}\right),
$$

where $g_{1}, \ldots, g_{\ell} \in G_{0}$ are the terms of $S$. Moreover, if $S_{1}, S_{2} \in \mathcal{F}\left(G_{0}\right)$ and $g_{1}, g_{2} \in G_{0}$, then $S_{1} \cdot S_{2} \in \mathcal{F}\left(G_{0}\right)$ has length $\left|S_{1}\right|+\left|S_{2}\right|, S_{1} \cdot g_{1} \in \mathcal{F}\left(G_{0}\right)$ has length $\left|S_{1}\right|+1, g_{1} g_{2} \in G$ is an element of $G$, but $g_{1} \cdot g_{2} \in \mathcal{F}\left(G_{0}\right)$ is a sequence of length 2 . If $g \in G_{0}, T \in \mathcal{F}\left(G_{0}\right)$, and $k \in \mathbb{N}_{0}$, then

$$
g^{[k]}=\underbrace{g \cdot \ldots \cdot g}_{k} \in \mathcal{F}\left(G_{0}\right) \quad \text { and } \quad T^{[k]}=\underbrace{T \cdot \ldots \cdot T}_{k} \in \mathcal{F}\left(G_{0}\right) .
$$

Let $S \in \mathcal{F}\left(G_{0}\right)$ be a sequence as in (3.1). Then we denote by

$$
\pi(S)=\left\{g_{\tau(1)} \ldots g_{\tau(\ell)} \in G \mid \tau \text { a permutation of }[1, \ell]\right\} \subset G \quad \text { and } \quad \Pi(S)=\bigcup_{\substack{T|S\\| T \mid \geq 1}} \pi(T) \subset G,
$$

the set of products and subsequence products of $S$, and it can easily be seen that $\pi(S)$ is contained in a $G^{\prime}$-coset. Note that $|S|=0$ if and only if $S=1_{\mathcal{F}\left(G_{0}\right)}$, and in that case we use the convention that $\pi(S)=\left\{1_{G}\right\}$. The sequence $S$ is called

- a product-one sequence if $1_{G} \in \pi(S)$, and

- product-one free if $1_{G} \notin \Pi(S)$. 
Definition 3.1. Let $G_{0} \subset G$ be a subset.

1. The submonoid

$$
\mathcal{B}\left(G_{0}\right)=\left\{S \in \mathcal{F}\left(G_{0}\right) \mid 1_{G} \in \pi(S)\right\} \subset \mathcal{F}\left(G_{0}\right)
$$

is called the monoid of product-one sequences, and $\mathcal{A}\left(G_{0}\right)=\mathcal{A}\left(\mathcal{B}\left(G_{0}\right)\right)$ denotes its set of atoms.

2. We call

$$
\mathrm{D}\left(G_{0}\right)=\sup \left\{|S| \mid S \in \mathcal{A}\left(G_{0}\right)\right\} \in \mathbb{N} \cup\{\infty\}
$$

the large Davenport constant of $G_{0}$ and

$$
\mathrm{d}\left(G_{0}\right)=\sup \left\{|S| \mid S \in \mathcal{F}\left(G_{0}\right) \text { is product-one free }\right\} \in \mathbb{N}_{0} \cup\{\infty\}
$$

the small Davenport constant of $G_{0}$.

Note that obviously the set $\mathcal{B}\left(G_{0}\right)$ is a multiplicatively closed subset of the commutative cancellative semigroup $\mathcal{F}\left(G_{0}\right)$ whence $\mathcal{B}\left(G_{0}\right)$ is indeed a monoid. Our object of interest is the monoid $\mathcal{B}(G)$ but for technical reasons we also need the submonoids $\mathcal{B}\left(G_{0}\right)$ for subsets $G_{0} \subset G$. The next lemma gathers some elementary properties. Its simple proof can be found in [9, Lemma 3.1].

Lemma 3.2. Let $G_{0} \subset G$ be a subset.

1. $\mathcal{B}\left(G_{0}\right) \subset \mathcal{F}\left(G_{0}\right)$ is a reduced finitely generated submonoid, $\mathcal{A}\left(G_{0}\right)$ is finite, and $\mathrm{D}\left(G_{0}\right) \leq|G|$.

2. Let $S \in \mathcal{F}(G)$ be product-one free.

(a) If $g_{0} \in \pi(S)$, then $g_{0}^{-1} \cdot S \in \mathcal{A}(G)$. In particular, $\mathrm{d}(G)+1 \leq \mathrm{D}(G)$.

(b) If $|S|=\mathrm{d}(G)$, then $\Pi(S)=G \backslash\left\{1_{G}\right\}$ and hence $\mathrm{d}(G)=\max \{|S|: S \in \mathcal{F}(G)$ with $\Pi(S)=$ $\left.G \backslash\left\{1_{G}\right\}\right\}$.

3. If $G$ is cyclic, then $\mathrm{d}(G)+1=\mathrm{D}(G)=|G|$.

The Davenport constant of finite abelian groups is a frequently studied invariant in Zero-sum Theory (for an overview see [18] and for recent progress [31, 5]). For the Davenport constant of nonabelian groups we refer to [17, 24, 10].

Lemma 3.3. Let $G_{0} \subset G$ be a subset. A submonoid $H \subset \mathcal{B}\left(G_{0}\right)$ is divisor-closed if and only if $H=\mathcal{B}\left(G_{1}\right)$ for a subset $G_{1} \subset G_{0}$.

Proof. If $G_{1} \subset G_{0}$, then clearly $\mathcal{B}\left(G_{1}\right) \subset \mathcal{B}\left(G_{0}\right)$ is a divisor-closed submonoid. Conversely, let $H \subset \mathcal{B}\left(G_{0}\right)$ be a divisor-closed submonoid. We set

$$
G_{1}=\bigcup_{B \in H} \operatorname{supp}(B)
$$

and obtain that $H \subset \mathcal{B}\left(G_{1}\right)$. We have to show that equality holds. Let $S=g_{1} \cdot \ldots \cdot g_{\ell} \in \mathcal{B}\left(G_{1}\right)$. Then for every $i \in[1, \ell]$ there is some $B_{i} \in H$ such that $g_{i} \in \operatorname{supp}\left(B_{i}\right)$. Then $B_{1} \cdot \ldots \cdot B_{\ell} \in H$ and $S$ divides $B_{1} \cdot \ldots \cdot B_{\ell}$, which implies that $S \in H$.

Proposition 3.4. The following statements are equivalent:

(a) $G$ is abelian.

(b) $\mathcal{B}(G)$ is a Krull monoid.

(c) $\mathcal{B}(G)$ is a transfer Krull monoid.

Proof. The implications (a) $\Rightarrow(\mathrm{b})$ and (b) $\Rightarrow$ (c) are well-known and immediate. Indeed, if $G$ is abelian, then the embedding $\mathcal{B}(G) \hookrightarrow \mathcal{F}(G)$ is a divisor homomorphism whence $\mathcal{B}(G)$ is Krull. By definition, every Krull monoid is a transfer Krull monoid.

(c) $\Rightarrow$ (a) Assume to the contrary that $G$ is not abelian but $\mathcal{B}(G)$ is a transfer Krull monoid. Thus there is a transfer homomorphism $\theta_{1}: \mathcal{B}(G) \rightarrow H$, where $H$ is a Krull monoid. Since there is a transfer homomorphism $\theta_{2}: H \rightarrow \mathcal{B}\left(G_{0}\right)$, where $G_{0} \subset \mathcal{C}(H)$ is a subset of the class group, and since the composition 
of transfer homomorphisms is a transfer homomorphism, we obtain a transfer homomorphism $\theta: \mathcal{B}(G) \rightarrow$ $\mathcal{B}\left(G_{0}\right)$, where $G_{0}$ is a subset of an abelian group.

Since $G$ is non-abelian, there exist $g, h \in G$ such that $g h \neq h g$. Consider the sequence

$$
U=g \cdot h \cdot g^{-1} \cdot\left(g h^{-1} g^{-1}\right) \in \mathcal{A}(G) .
$$

From [18, Proposition 3.2.3], we have that $\theta(U) \in \mathcal{A}\left(G_{0}\right)$, say $\theta(U)=a_{1} \cdot \ldots \cdot a_{\ell}$, where $a_{i} \in G_{0}$ for all $i \in[1, \ell]$. Let $m=$ ord $\left(h g h^{-1} g^{-1}\right) \in \mathbb{N}$. Then

$$
U^{[m]}=\left(g \cdot g^{-1}\right)^{[m]} \cdot\left(h \cdot\left(g h^{-1} g^{-1}\right)\right)^{[m]},
$$

and hence it follows that

$$
\begin{aligned}
\left(a_{1} \cdot \ldots \cdot a_{\ell}\right)^{[m]} & =\theta\left(U^{[m]}\right) \\
& =\left(\theta\left(g \cdot g^{-1}\right)\right)^{[m]} \cdot \theta\left(\left(h \cdot\left(g h^{-1} g^{-1}\right)\right)^{[m]}\right) .
\end{aligned}
$$

Since $\mathcal{B}\left(G_{0}\right) \hookrightarrow \mathcal{F}\left(G_{0}\right)$ is a divisor homomorphism (and so $\mathcal{B}\left(G_{0}\right)$ is root closed), the fact that $\left(\theta\left(g \cdot g^{-1}\right)\right)^{[m]}$ divides $\left(a_{1} \cdot \ldots \cdot a_{\ell}\right)^{[m]}$ in $\mathcal{B}\left(G_{0}\right)$ implies that $\theta\left(g \cdot g^{-1}\right)$ divides $a_{1} \cdot \ldots \cdot a_{\ell}$ in $\mathcal{B}\left(G_{0}\right)$. Since $\theta\left(g \cdot g^{-1}\right)$ and $a_{1}$. $\ldots \cdot a_{\ell} \in \mathcal{A}\left(G_{0}\right)$, it follows that $\theta\left(g \cdot g^{-1}\right)=a_{1} \cdot \ldots \cdot a_{\ell}=\theta(U)$. Thus $\theta\left(\left(h \cdot\left(g h^{-1} g^{-1}\right)\right)^{[m]}\right)=1_{\mathcal{F}\left(G_{0}\right)}$, a contradiction.

Clearly, if $|G| \leq 2$, then $\mathcal{B}(G)$ is factorial whence it is both a Krull monoid (with trivial class group) and a C-monoid (with trivial class semigroup). In order to avoid trivial case distinctions, we exclude this case whenever convenient. By Proposition 3.4, $\mathcal{B}(G)$ is not Krull when $G$ is not abelian. The next proposition reveals that $\mathcal{B}(G)$ is a $\mathrm{C}$-monoid in all cases and determines the class group of its complete integral closure.

Proposition 3.5. Suppose that $|G| \geq 3$.

1. If $G_{0} \subset G$ is a subset, then $\mathcal{B}\left(G_{0}\right) \subset \mathcal{F}\left(G_{0}\right)$ is cofinal and $\mathcal{B}\left(G_{0}\right)$ is a C-monoid.

2. The embedding $\widehat{\mathcal{B}(G)} \hookrightarrow \mathcal{F}(G)$ is a divisor theory and the map

$$
\begin{aligned}
\Phi: \mathcal{C}(\widehat{\mathcal{B}(G)})=\mathrm{q}(\mathcal{F}(G)) / \mathrm{q}(\mathcal{B}(G)) & \longrightarrow G / G^{\prime} \\
S \mathrm{q}(\mathcal{B}(G)) & \longmapsto g G^{\prime},
\end{aligned}
$$

where $S \in \mathcal{F}(G)$ and $g \in \pi(S)$, is an isomorphism. Clearly, $\vee_{g}(\mathcal{B}(G))=\mathbb{N}_{0}$ for all $g \in G$.

3. There is an epimorphism $\mathcal{C}(\mathcal{B}(G), \mathcal{F}(G)) \rightarrow G / G^{\prime}$.

Proof. 1. and 2. Let $G_{0} \subset G$. If $S=g_{1} \cdot \ldots \cdot g_{\ell} \in \mathcal{F}\left(G_{0}\right)$ and $g \in \pi(S)$, then $S^{\prime}=g^{-1} \cdot S \in \mathcal{B}\left(G_{0}\right), S \mid S^{\prime}$, and hence $\mathcal{B}\left(G_{0}\right) \subset \mathcal{F}\left(G_{0}\right)$ is cofinal. Let $k \in \mathbb{N}_{0}$. If $g \in G$ with ord $(g)>2$, then $U=g \cdot g^{-1} \in \mathcal{B}(G)$, $\mathrm{v}_{g}\left(U^{[k]}\right)=k$. If $g=1_{G}$, then $\mathrm{v}_{g}\left(g^{[k]}\right)=k$. If $\operatorname{ord}(g)=2$, then there is an $h \in G \backslash\left\{1_{G}, g\right\}$ and $U=g \cdot h \cdot(g h)^{-1} \in \mathcal{B}(G)$ and $\vee_{g}\left(U^{[k]}\right)=k$. Thus in all cases we have $\vee_{g}(\mathcal{B}(G))=\mathbb{N}_{0}$.

It is easy to check that $\Phi$ is an isomorphism (details can be found in [9, Theorem 3.2]). Since $\mathcal{C}(\widehat{\mathcal{B}(G)})$ is finite, $\mathcal{C}\left(\widehat{\mathcal{B}\left(G_{0}\right)}\right)$ is finite and since $\mathcal{B}\left(G_{0}\right)$ is finitely generated, it is a C-monoid by [19, Proposition 4.8].

3. This follows from 2 . and from Lemma $2.2,2$.

We start with the study of the class semigroup and recall that, by definition, for two sequences $S, S^{\prime} \in$ $\mathcal{F}(G)$ the following statements are equivalent:

- $S \sim \mathcal{B}(G) S^{\prime}$.

- For all $T \in \mathcal{F}(G)$, we have $S \cdot T \in \mathcal{B}(G)$ if and only if $S^{\prime} \cdot T \in \mathcal{B}(G)$.

- For all $T \in \mathcal{F}(G)$, we have $1_{G} \in \pi(S \cdot T)$ if and only if $1_{G} \in \pi\left(S^{\prime} \cdot T\right)$.

If $S=g_{1} \cdot \ldots \cdot g_{\ell} \in \mathcal{B}(G)$ such that $1_{G}=g_{1} \ldots g_{\ell}$, then $1_{G}=g_{i} \ldots g_{\ell} g_{1} \ldots g_{i-1}$ for every $i \in[1, \ell]$. We will use this simple fact without further mention. Moreover, $\sim$ means $\sim_{\mathcal{B}(G)}$ and we write $[S]=[S]_{\mathcal{B}(G)}^{\mathcal{F}(G)}$. 
Lemma 3.6. Let $S \in \mathcal{F}(G)$.

1. If $S^{\prime} \in \mathcal{F}(G)$ such that $S \sim S^{\prime}$, then $\pi(S)=\pi\left(S^{\prime}\right)$.

2. Let $S^{\prime} \in \mathcal{F}(G)$. In the following cases, $S \sim S^{\prime}$ if and only if $\pi(S)=\pi\left(S^{\prime}\right)$ :

(a) $S$ and $S^{\prime}$ are sequences over the center of $G$.

(b) There is $g \in \pi(S)$ such that $\pi(S)=g G^{\prime}$.

3. If $g, h \in G$ with $g \neq h$, then $g \nsim h$. In particular, $|G| \leq|\mathcal{C}(\mathcal{B}(G), \mathcal{F}(G))|$.

4. If $g \in \mathrm{Z}(G)$ and $h \in G$, then $g \cdot h \sim g h$.

5. $|\pi(S)|=1$ if and only if $\langle\operatorname{supp}(S)\rangle$ is abelian.

Proof. 1. Suppose that $S^{\prime} \in \mathcal{F}(G)$ with $S \sim S^{\prime}$. Then for every $g \in G$ we obtain that

$$
g \in \pi(S) \quad \Leftrightarrow \quad g^{-1} \cdot S \in \mathcal{B}(G) \quad \Leftrightarrow \quad g^{-1} \cdot S^{\prime} \in \mathcal{B}(G) \quad \Leftrightarrow \quad g \in \pi\left(S^{\prime}\right),
$$

which implies that $\pi(S)=\pi\left(S^{\prime}\right)$.

2. From 1., it remains to verify the reverse implication. Suppose that $\pi(S)=\pi\left(S^{\prime}\right)$.

(a) Since $S, S^{\prime} \in \mathcal{F}(\mathrm{Z}(G))$, we have $|\pi(S)|=\left|\pi\left(S^{\prime}\right)\right|=1$, say $\pi(S)=\pi\left(S^{\prime}\right)=\{g\}$. Then for every $T \in \mathcal{F}(G)$ we have

which implies that $S \sim S^{\prime}$.

$$
\pi(S \cdot T)=g \pi(T) \quad \text { and } \quad \pi\left(S^{\prime} \cdot T\right)=g \pi(T),
$$

(b) Suppose that there is a $g \in \pi(S)$ such that $\pi(S)=g G^{\prime}$. Let $T \in \mathcal{F}(G)$ such that $T \cdot S \in \mathcal{B}(G)$. Then we have

$$
\pi(T) \pi(S) \subset \pi(T \cdot S) \subset G^{\prime} .
$$

Since $\pi(S)=g G^{\prime}$, there are $t \in \pi(T)$ and $e \in G^{\prime}$ such that $t g=e$. Hence we obtain that

$$
1_{G}=e e^{-1}=t g e^{-1} \in \pi(T) \pi(S)=\pi(T) \pi\left(S^{\prime}\right) \subset \pi\left(T \cdot S^{\prime}\right) .
$$

It follows that $T \cdot S^{\prime} \in \mathcal{B}(G)$. By symmetry, we infer that $S \sim S^{\prime}$.

3. Let $g, h \in G$. Then $g, h \in \mathcal{F}(G)$ and if $g \sim h$, then 1 . implies that $\{g\}=\pi(g)=\pi(h)=\{h\}$. Therefore, $g \neq h$ implies that $g \nsim h$, and hence we obtain that $|G| \leq|\mathcal{C}(\mathcal{B}(G), \mathcal{F}(G))|$.

4. This follows from the fact that $g \in \mathrm{Z}(G)$.

5. Obvious.

Lemma 3.7. Let $S \in \mathcal{F}(G)$.

1. If $[S]$ is an idempotent of $\mathcal{C}(\mathcal{B}(G), \mathcal{F}(G))$, then $\pi(S) \subset G^{\prime}$ is a subgroup.

2. $\left[1_{\mathcal{F}(G)}\right]=\mathcal{B}(\mathrm{Z}(G))$. In particular, if $g \in \mathbf{Z}(G)$, then $g^{[\operatorname{ord}(g)]} \sim 1_{\mathcal{F}(G)}$.

3. $[S]$ is the smallest idempotent in $\mathrm{E}(\mathcal{C}(\mathcal{B}(G), \mathcal{F}(G)))$ (with respect to the Rees order) if and only if $\pi(S)=G^{\prime}$.

Proof. 1. Suppose that $[S]$ is an idempotent of $\mathcal{C}(\mathcal{B}(G), \mathcal{F}(G))$. Then $[S]=\left[S^{[2]}\right]$ whence $S \sim S^{[2]}$, and Lemma 3.6, 1 implies that

Thus $\pi(S) \subset G^{\prime}$ is a subgroup.

$$
\pi(S) \pi(S) \subset \pi\left(S^{[2]}\right)=\pi(S)
$$

2. Suppose that $S \in \mathcal{B}(\mathrm{Z}(G))$. Then $\pi(S)=\left\{1_{G}\right\}$, and for all $T \in \mathcal{F}(G)$ we have

$$
\pi(S \cdot T)=\pi(T)=\pi\left(1_{\mathcal{F}(G)} \cdot T\right),
$$

whence $S \sim 1_{\mathcal{F}(G)}$.

Conversely, suppose that $S \sim 1_{\mathcal{F}(G)}$. Then $S \in \mathcal{B}(G)$, and we set $S=g_{1} \cdot \ldots \cdot g_{\ell}$ such that $g_{1} \ldots g_{\ell}=1_{G}$. Assume to the contrary that there is an $i \in[1, \ell]$ such that $g_{i} \notin \mathrm{Z}(G)$, say $i=1$. Then there is an element $h \in G$ such that $h g_{1} \neq g_{1} h$. Then $T=\left(h g_{1}\right) \cdot\left(h^{-1} g_{1}^{-1}\right) \in \mathcal{F}(G) \backslash \mathcal{B}(G)$, but 


$$
1_{G}=g_{1}\left(h g_{1}\right)\left(g_{2} \ldots g_{\ell}\right)\left(h^{-1} g_{1}^{-1}\right) \in \pi(T \cdot S) .
$$

Since $S \sim 1_{\mathcal{F}(G)}$, we infer that $1_{G} \in \pi\left(T \cdot 1_{\mathcal{F}(G)}\right)=\pi(T)$, a contradiction.

In particular, if $g \in \mathrm{Z}(G)$, then $g^{[\operatorname{ord}(g)]} \in \mathcal{B}(\mathrm{Z}(G))$ and hence $g^{\operatorname{lord}(g)]} \sim 1_{\mathcal{F}(G)}$.

3. First, we suppose that $\pi(S)=G^{\prime}$. Then $[S]$ is an idempotent by Lemma 3.6]2, and it remains to verify that $[S]$ is the smallest idempotent of $\mathcal{C}(\mathcal{B}(G), \mathcal{F}(G))$. Let $S^{\prime} \in \mathcal{F}(G)$ such that $\left[S^{\prime}\right]$ is an idempotent. We have to show that $S \sim S \cdot S^{\prime}$. Since $\pi\left(S^{\prime}\right) \subset G^{\prime}$ is a subgroup by 1 ., $S, S^{\prime} \in \mathcal{B}(G)$, and since $\pi\left(S \cdot S^{\prime}\right)$ is a $G^{\prime}$-coset, it follows that

$$
G^{\prime} \subset \pi(S) \pi\left(S^{\prime}\right) \subset \pi\left(S \cdot S^{\prime}\right) \subset G^{\prime} \text { whence } G^{\prime}=\pi\left(S \cdot S^{\prime}\right) .
$$

Again Lemma 3.6.2 implies that $S \sim S \cdot S^{\prime}$.

To show the converse, suppose that $[S]$ is the smallest idempotent. We construct an element $S^{\prime} \in \mathcal{F}(G)$ such that $\pi\left(S^{\prime}\right)=G^{\prime}$. Then the proof above shows that $\left[S^{\prime}\right]$ is the smallest idempotent whence $S \sim S^{\prime}$ and $\pi(S)=\pi\left(S^{\prime}\right)=G^{\prime}$. We set $G^{\prime}=\left\{g_{1}, \ldots, g_{n}\right\}$, and for each $i \in[1, n]$ we set

$$
g_{i}=\prod_{\nu=1}^{k_{i}} a_{i, \nu} b_{i, \nu} a_{i, \nu}^{-1} b_{i, \nu}^{-1}, \quad \text { where } k_{i} \in \mathbb{N} \text { and all } \quad a_{i, \nu}, b_{i, \nu} \in G
$$

and define

$$
S_{i}=\underset{\nu \in\left[1, k_{i}\right]}{\bullet}\left(a_{i, \nu} \cdot b_{i, \nu} \cdot a_{i, \nu}^{-1} \cdot b_{i, \nu}^{-1}\right) \in \mathcal{B}(G) .
$$

Then obviously $\pi\left(S_{1} \cdot \ldots \cdot S_{n}\right)=G^{\prime}$.

\section{Theorem 3.8.}

1. Suppose that $G / G^{\prime}=\left\{g_{0} G^{\prime}, \ldots, g_{k} G^{\prime}\right\}$, and for each $i \in[0, k]$ let $S_{i} \in \mathcal{F}(G)$ such that $\pi\left(S_{i}\right)=g_{i} G^{\prime}$. Then the map

$$
\begin{aligned}
G / G^{\prime} & \rightarrow\left\{\left[S_{i}\right] \mid i \in[0, k]\right\}=\mathcal{C} \subset \mathcal{C}(\mathcal{B}(G), \mathcal{F}(G)) \\
g_{i} G^{\prime} & \mapsto\left[S_{i}\right]
\end{aligned}
$$

is a group isomorphism. Thus $\mathcal{C}(\mathcal{B}(G), \mathcal{F}(G))$ contains a subgroup isomorphic to the class group of $\widehat{\mathcal{B}(G)}$. Moreover, for any $i \in[0, k]$ and for any $S \in \mathcal{F}(G)$, we have $\left[S \cdot S_{i}\right] \in \mathcal{C}$.

2. The map

is a group isomorphism.

$$
\begin{aligned}
\mathrm{Z}(G) & \rightarrow \mathcal{C}(\mathcal{B}(G), \mathcal{F}(G))^{\times} \\
g & \mapsto[g]
\end{aligned}
$$

Proof. 1. We first verify the existence of such sequences $S_{0}, \ldots, S_{k}$. Since $\mathcal{C}(\mathcal{B}(G), \mathcal{F}(G))$ is finite, the set $\mathrm{E}(\mathcal{C}(\mathcal{B}(G), \mathcal{F}(G)))$ has the smallest element, say $[S]$. For each $i \in[0, k]$, we set $S_{i}=g_{i} \cdot S$. Since $\pi(S)=G^{\prime}$ by Lemma $3.7,3$, it follows that for each $i \in[0, k]$

$$
g_{i} G^{\prime} \subset \pi\left(g_{i} \cdot S\right) \subset g_{i} G^{\prime}, \text { whence } \pi\left(S_{i}\right)=\pi\left(g_{i} \cdot S\right)=g_{i} G^{\prime} .
$$

We now use Lemma 3.6. 2. If $i, j \in[0, k]$, then $g_{i} g_{j} \in \pi\left(S_{i}\right) \pi\left(S_{j}\right) \subset \pi\left(S_{i} \cdot S_{j}\right)$, and hence $\pi\left(S_{i} \cdot S_{j}\right)=$ $g_{i} g_{j} G^{\prime}=g_{\ell} G^{\prime}=\pi\left(S_{\ell}\right)$ for some $\ell \in[0, k]$. Thus it follows that $\mathcal{C}$ is a subgroup of the class semigroup and the given map is an isomorphism. By Proposition $3.5,2, G / G^{\prime}$ is isomorphic to the class group of $\widehat{\mathcal{B}(G)}$.

Moreover, let $S \in \mathcal{F}(G), g \in \pi(S)$, and $i \in[0, k]$. Then

$$
g g_{i} G^{\prime}=g \pi\left(S_{i}\right) \subset \pi(S) \pi\left(S_{i}\right) \subset \pi\left(S \cdot S_{i}\right),
$$

whence $\pi\left(S \cdot S_{i}\right)=g g_{i} G^{\prime}=g_{j} G^{\prime}=\pi\left(S_{j}\right)$ for some $j \in[0, k]$. Again by Lemma 3.6] 2 , we have $S \cdot S_{i} \sim S_{j}$, and thus $\left[S \cdot S_{i}\right] \in \mathcal{C}$. 
2. Let $S \in \mathcal{F}(G)$ such that $[S] \in \mathcal{C}(\mathcal{F}(G), \mathcal{B}(G))$ is invertible. Then there is an $S^{\prime} \in \mathcal{F}(G)$ such that

$$
0_{\mathcal{C}(\mathcal{F}(G), \mathcal{B}(G))}=\left[1_{\mathcal{F}(G)}\right]=[S]+\left[S^{\prime}\right]=\left[S \cdot S^{\prime}\right],
$$

whence $S \cdot S^{\prime} \sim 1_{\mathcal{F}(G)}$. Then Lemma 3.7,2 implies that $S \cdot S^{\prime} \in \mathcal{B}(\mathrm{Z}(G))$ whence $S, S^{\prime} \in \mathcal{F}(\mathrm{Z}(G))$. If $g \in \pi(S)$, then Lemma 3.6.2 implies that $S \sim g$. This proves that the given map is well-defined and surjective. Lemma 3.6 (items 3 and 4 ) shows that the map is a monomorphism.

Our next goal is a detailed investigation of the case where $\left|G^{\prime}\right|=2$. We derive a couple of special properties which do not hold in general (Theorem 3.10 and Remark 3.11).

Lemma 3.9. Suppose that $\left|G^{\prime}\right|=2$, and let $g \in G$ with $\operatorname{ord}(g)=n$.

1. We have $g \sim g^{[n+1]}$, and hence $\left[g^{[n]}\right] \in \mathcal{C}(\mathcal{B}(G), \mathcal{F}(G))$ is idempotent.

2. If $k \in[1, n]$ is odd and $g^{k} \notin \mathrm{Z}(G)$, then $g^{[k]} \sim g^{k}$.

3. If $g, h \in G \backslash \mathrm{Z}(G)$ with $g h=h g$, then $g \cdot h \nsim$ gh provided that one of the following conditions holds:

(a) $g h \in \mathrm{Z}(G)$.

(b) There is $g_{0} \in G$ such that $g_{0} g \neq g g_{0}$ and $g_{0} h \neq h g_{0}$.

Proof. 1. Let $T \in \mathcal{F}(G)$. Since $g^{n}=1_{G}, 1_{G} \in \pi(g \cdot T)$ implies that $1_{G} \in \pi\left(g^{[n+1]} \cdot T\right)$. Conversely, suppose that $1_{G} \in \pi\left(g^{[n+1]} \cdot T\right)$. If every element in $\operatorname{supp}(T)$ commutes with $g$, then

$$
\pi\left(g^{[n+1]} \cdot T\right)=\pi(g \cdot T) \text { and hence } \quad 1_{G} \in \pi(g \cdot T) .
$$

Now suppose that there is at least one $h \in \operatorname{supp}(T)$ such that $h g \neq g h$. Then $\pi(g \cdot T)$ has at least two elements. Since $\left|G^{\prime}\right|=2$ and

$$
\pi(g \cdot T) \subset \pi\left(g^{[n+1]} \cdot T\right) \subset G^{\prime}
$$

we obtain that $\pi(g \cdot T)=G^{\prime}$ and hence $1_{G} \in \pi(g \cdot T)$. Thus $[g]=\left[g^{[n+1]}\right]$ and thus

$$
\left[g^{[n]}\right]+\left[g^{[n]}\right]=\left[g^{[2 n]}\right]=\left[g^{[n+1]} \cdot g^{[n-1]}\right]=\left[g^{[n]}\right] .
$$

2. Let $k \in[1, n]$ be odd, $g^{k} \notin \mathrm{Z}(G)$, and $T \in \mathcal{F}(G)$. If $1_{G} \in \pi\left(g^{k} \cdot T\right)$, then $1_{G} \in \pi\left(g^{[k]} \cdot T\right)$. Conversely, suppose that $1_{G} \in \pi\left(T \cdot g^{[k]}\right)$. If $h g=g h$ for all $h \in \operatorname{supp}(T)$, then obviously $1_{G} \in \pi\left(g^{k} \cdot T\right)$.

Suppose there is an element $h \in \operatorname{supp}(T)$ such that $h g \neq g h$. Then $\pi\left(T \cdot g^{[k]}\right)=G^{\prime}$. We set $T=h_{1} \cdot \ldots \cdot h_{\ell}$ with $h_{1}=h$ and consider the elements

$$
g_{0}=h_{1} g^{k} h_{2} \ldots h_{\ell} \quad \text { and } \quad g_{0}^{(1)}=g h_{1} g^{k-1} h_{2} \ldots h_{\ell} \quad \text { in } G^{\prime} .
$$

Then $G^{\prime}=\left\{g_{0}, g_{0}^{(1)}\right\}$. If $g_{0}=1$, then we are done. Suppose that $g_{0}^{(1)}=1_{G}$. Then

$$
g_{0}^{(2)}=g g h_{1} g^{k-2} h_{2} \ldots h_{\ell} \neq g_{0}^{(1)}
$$

whence $g_{0}^{(2)}=g_{0}$, and $h g^{2}=g^{2} h$. Thus we obtain

$$
1_{G}=g_{0}^{(1)}=g_{0}^{(3)}=\ldots=g_{0}^{(k)}=g^{k} h_{1} \ldots h_{\ell} \in \pi\left(T \cdot g^{k}\right) .
$$

3. Let $g, h \in G \backslash \mathrm{Z}(G)$ with $g h=h g$.

(a) Suppose that $g h \in \mathbf{Z}(G)$ and assume to the contrary that $g \cdot h \sim g h$. By Lemma 3.7 2, we infer that

$$
(g \cdot h)^{[\operatorname{ord}(g h)]} \sim g h^{[\operatorname{ord}(g h)]} \sim 1_{\mathcal{F}(G)} .
$$

a contradiction to $\left[1_{\mathcal{F}(G)}\right]=\mathcal{B}(\mathrm{Z}(G))$.

(b) Let $g_{0} \in G$ such that $g_{0} g \neq g g_{0}$ and $g_{0} h \neq h g_{0}$. If

$$
T=\left(g^{-1} g_{0}\right) \cdot\left(h^{-1} g_{0}^{-1}\right) \in \mathcal{F}(G),
$$

then $1_{G} \notin \pi(T \cdot(g h))$ but $1_{G}=g\left(g^{-1} g_{0}\right) h\left(h^{-1} g_{0}^{-1}\right) \in \pi(T \cdot g \cdot h)$. This shows that $g \cdot h \nsim g h$. 
It is well-known that a finitely generated monoid is Krull if and only if it is root-closed. Thus if $G$ is non-abelian, then $\mathcal{B}(G)$ is not root-closed by Proposition 3.4. The next result shows that it is seminormal if $\left|G^{\prime}\right|=2$. An element $c$ of a semigroup is called a regular if $c$ lies in a subgroup of the semigroup, and the semigroup is called Clifford if every element is regular.

Theorem 3.10. Suppose that $\left|G^{\prime}\right|=2$.

1. $\mathcal{B}(G)$ is seminormal.

2. $\mathcal{C}(\mathcal{B}(G), \mathcal{F}(G))$ is a Clifford semigroup. In particular, if $S \in \mathcal{F}(G)$ with $\pi(S)=\{g\}$, then $[S]$ generates a cyclic subgroup of order $\operatorname{ord}(g)$.

3. $|\mathcal{C}(\mathcal{B}(G), \mathcal{F}(G))| \leq|\mathrm{Z}(G)|+\prod_{g \in G \backslash \mathrm{Z}(G)} \operatorname{ord}(g)$.

Proof. 1. Let $S \in \mathrm{q}(\mathcal{B}(G))$ such that $S^{[2]}, S^{[3]} \in \mathcal{B}(G)$. Since $S \in \mathrm{q}(\mathcal{F}(G))$ with $S^{[2]}, S^{[3]} \in \mathcal{F}(G)$, we have that $S \in \mathcal{F}(G)$. Let $g \in \pi(S)$. Since $S^{[2]}, S^{[3]} \in \mathcal{B}(G)$, it follows that

$$
g^{2} \in \pi\left(S^{[2]}\right) \subset G^{\prime} \quad \text { and } \quad g^{3} \in \pi\left(S^{[3]}\right) \subset G^{\prime} .
$$

Since $G^{\prime}$ is a subgroup of $G$, we obtain $g \in G^{\prime}$. If $g=1_{G}$, then we are done. Suppose that $g \neq 1_{G}$. Then $G^{\prime}=\left\{1_{G}, g\right\}$. If each two elements in $\operatorname{supp}(S)$ would commute, then

$$
\pi(S)=\{g\}, \quad \pi\left(S^{[2]}\right)=\left\{g^{2}\right\}, \text { and } \pi\left(S^{[3]}\right)=\left\{g^{3}\right\},
$$

which implies $g^{2}=1_{G}=g^{3}$ and $g=1_{G}$, a contradiction. Thus $\operatorname{supp}(S)$ contains two elements which do not commute, say $S=g_{1} \cdot \ldots \cdot g_{\ell}, g_{1} g_{2} \neq g_{2} g_{1}$, and $g=g_{1} \ldots g_{\ell}$. Then $1_{G}=g_{2} g_{1} g_{3} \ldots g_{\ell} \in \pi(S)$.

2. Let $S \in \mathcal{F}(G)$. If $\pi(S)$ has two elements, then $[S]$ lies in the group given in Theorem 3.8.1. Suppose that $\pi(S)$ has only one element, say $\pi(S)=\{g\}$ and $\operatorname{ord}(g)=n$. We assert that $S \sim S^{[n+1]}$. Suppose this holds true. Then $\left\{[S], \ldots,\left[S^{[n]}\right]\right\}$ is a cyclic subgroup of the class semigroup, and hence the assertion follows. Let $m \in[1, n]$ and $\left[S^{[m]}\right]$ the identity element of the subgroup. Then it is an idempotent of the class semigroup, and Lemma 3.7.1 shows that $\pi\left(S^{[m]}\right)=\left\{g^{m}\right\} \subset G^{\prime}$ is a subgroup. This implies $m=n$.

Thus it remains to show that $S \sim S^{[n+1]}$. To do so, let $T \in \mathcal{F}(G)$ be given. Since $S^{[n]} \in \mathcal{B}(G)$,

$$
1_{G} \in \pi(T \cdot S) \quad \text { implies that } \quad 1_{G} \in \pi\left(T \cdot S^{[n+1]}\right) .
$$

Conversely, suppose that $1_{G} \in \pi\left(T \cdot S^{[n+1]}\right)$. If every element of $\operatorname{supp}(T)$ commutes with every element of $\operatorname{supp}(S)$, then $\pi\left(T \cdot S^{[n+1]}\right)=\pi(T \cdot S)$ and thus $1_{G} \in \pi(T \cdot S)$. If there are $t \in \operatorname{supp}(T)$ and $s \in \operatorname{supp}(S)$ such that $t s \neq s t$, then $|\pi(T \cdot S)| \geq 2$ and hence $1_{G} \in \pi(T \cdot S)$.

3. We set $G=\left\{g_{1}, \ldots, g_{n}\right\}$ and $\mathrm{Z}(G)=\left\{g_{1}, \ldots, g_{k}\right\}$ for some $k \in[1, n]$. Let $S \in \mathcal{F}(G)$. Then we can write $S$ of the form

$$
S=g_{1}^{\left[k_{1} \operatorname{ord}\left(g_{1}\right)+r_{1}\right]} \cdot \ldots \cdot g_{n}^{\left[k_{n} \operatorname{ord}\left(g_{n}\right)+r_{n}\right]},
$$

where $k_{1}, \ldots, k_{n} \in \mathbb{N}_{0}$ and $r_{i} \in\left[1, \operatorname{ord}\left(g_{i}\right)\right]$ for each $i \in[1, n]$. By Lemma [3.9]1, we have

$$
S \sim g_{1}^{\left[r_{1}\right]} \cdot \ldots \cdot g_{n}^{\left[r_{n}\right]} .
$$

By Lemma 3.6.4., $g_{1}^{\left[r_{1}\right]} \cdot \ldots \cdot g_{k}^{\left[r_{k}\right]} \sim g_{0}$, where $g_{0}=g_{1}^{r_{1}} \ldots g_{k}^{r_{k}} \in \mathbf{Z}(G)$. Thus

$$
S \sim g_{0} \cdot g_{k+1}^{\left[r_{k+1}\right]} \cdot \ldots \cdot g_{n}^{\left[r_{n}\right]}
$$

and hence the assertion follows.

\section{Remark 3.11.}

1. Suppose that $\left|G^{\prime}\right|=2$. Let $g, h \in G$ be distinct with $\operatorname{ord}(g)=n$ and $\operatorname{ord}(h)=m$. By Theorem 3.10, $2,[g] \subset \mathcal{C}(\mathcal{B}(G), \mathcal{F}(G))$ generates a cyclic subgroup of order $n$ and $[h]$ generates a cyclic subgroup of order $m$. 
Suppose that $\langle[g]\rangle \cap\langle[h]\rangle \neq \emptyset$. Then there are $i \in[1, n]$ and $j \in[1, m]$ such that $g^{[i]} \sim h^{[j]}$. Let $m_{j}=\operatorname{ord}\left(h^{j}\right)=\frac{m}{\operatorname{gcd}(j, m)}$. Since $\left[h^{[m]}\right]$ is an idempotent, we have

$$
g^{\left[i m_{j}\right]} \sim h^{[m]} .
$$

By Lemma 3.6. $1, i m_{j}=k n$ for some $k \in \mathbb{N}$. Since $\left[g^{[n]}\right]$ is also idempotent, we have

$$
g^{[n]} \sim h^{[m]} .
$$

Again by Lemma 3.6 1 , we obtain $g h=h g$ because $\mathcal{B}(G)$-equivalence is a congruence relation on $\mathcal{F}(G)$.

It follows that if $g h \neq h g$, then $\langle[g]\rangle \cap\langle[h]\rangle=\emptyset$, and for each $i \in[1, n]$ and $j \in[1, m]$,

$$
\left[g^{[i]}\right]+\left[h^{[j]}\right]=\left[g^{[i]} \cdot h^{[j]}\right] \in \mathcal{C},
$$

where $\mathcal{C}$ is the group given in Theorem 3.81 .

2. Suppose that $\mathcal{B}(G)$ is seminormal and let $G_{0} \subset G$ be a subset. Let $S \in \mathrm{q}\left(\mathcal{B}\left(G_{0}\right)\right)$ such that $S^{[2]}, S^{[3]} \in \mathcal{B}\left(G_{0}\right)$. Since $\mathcal{B}(G)$ is seminormal, it follows that $S \in \mathcal{B}(G)$ and hence $S \in \mathcal{B}(G) \cap \mathrm{q}\left(\mathcal{B}\left(G_{0}\right)\right)=$ $\mathcal{B}\left(G_{0}\right)$. Thus $\mathcal{B}\left(G_{0}\right)$ is seminormal.

3. Let $D_{2 n}=\langle a, b\rangle=\left\{1_{G}, a, \ldots, a^{n-1}, b, a b, \ldots, a^{n-1} b\right\}$ be the dihedral group with $n \equiv 3 \bmod 4$, where $\operatorname{ord}(a)=n, \operatorname{ord}(b)=2$, and $a^{k} b a^{\ell} b=a^{k-\ell}$ for all $k, \ell \in \mathbb{Z}$. Then

$$
S=a^{\left[\frac{n-1}{2}\right]} \cdot b^{[2]} \in \mathrm{q}\left(\mathcal{B}\left(D_{2 n}\right)\right) \backslash \mathcal{B}\left(D_{2 n}\right) \text {, but } S^{[2]}, S^{[3]} \in \mathcal{B}\left(D_{2 n}\right) .
$$

Thus if $G$ contains $D_{2 n}$ as a subgroup, then 2. shows that $\mathcal{B}(G)$ is not seminormal.

\section{Examples of Class Semigroups for Non-abelian Groups of Small order}

In this section, we discuss the three smallest non-abelian groups and provide a complete description of the class semigroup. Among others we will see that the monoid of product-one sequences over the dihedral group with 6 elements is not seminormal and the associated class semigroup is not Clifford.

Example 4.1. Let $G=Q_{8}=\{E, I, J, K,-E,-I,-J,-K\}$ be the quaternion group with the relations

$$
I J=-J I=K, \quad J K=-K J=I, \quad K I=-I K=J, \quad \text { and } \quad I J K=-E .
$$

Then $\mathrm{Z}(G)=G^{\prime}=\{E,-E\}$ and $G / G^{\prime} \simeq C_{2} \oplus C_{2}$. Furthermore, $\mathrm{d}(G)=4$ and $\mathrm{D}(G)=6$ by [17, Theorem 1.1].

Let $S \in \mathcal{F}(G)$. If $|\pi(S)|=2$, then, by Theorem 3.8,1, $S$ is $\mathcal{B}(G)$-equivalent to an element in the group $\mathcal{C}$ which is isomorphic to $G / G^{\prime}$. We only consider the case $|\pi(S)|=1$. By Lemma 3.6.5, $\langle\operatorname{supp}(S)\rangle$ is an abelian subgroup of $G$. Suppose that $S \in \mathcal{F}(\langle I\rangle)$. Then $S$ has of the form

$$
S=E^{\left[n_{1}\right]} \cdot I^{\left[n_{2}\right]} \cdot(-E)^{\left[n_{3}\right]} \cdot(-I)^{\left[n_{4}\right]},
$$

where $n_{1}, \ldots, n_{4} \in \mathbb{N}_{0}$. By Lemma 3.6.4 and Lemma 3.9 (items 1 and 2), we have

$$
S \sim I^{[n]} \text { for some } n \in[1,4] .
$$

By symmetry, we obtain the same results in the case $S \in \mathcal{F}(\langle J\rangle)$ and $S \in \mathcal{F}(\langle K\rangle)$. Moreover, If $S \in \mathcal{F}(\langle-E\rangle)$, then, by Theorem 3.8 2, $S$ is $\mathcal{B}(G)$-equivalent to an element in the group of units of the class semigroup $\mathcal{C}(\mathcal{B}(G), \mathcal{F}(G))$ which is isomorphic to $\mathrm{Z}(G)$. It follows that

$$
|\mathcal{C}(\mathcal{B}(G), \mathcal{F}(G))|=18 \text {. }
$$

Figure 1 presents the subgroup lattice of the class semigroup. Note that the subgroup lattice of $G$ is not preserved in the class semigroup $\mathcal{C}(\mathcal{B}(G), \mathcal{F}(G))$. Furthermore, observe that the bottom elements in the lattice are all idempotent elements of the class semigroup. 


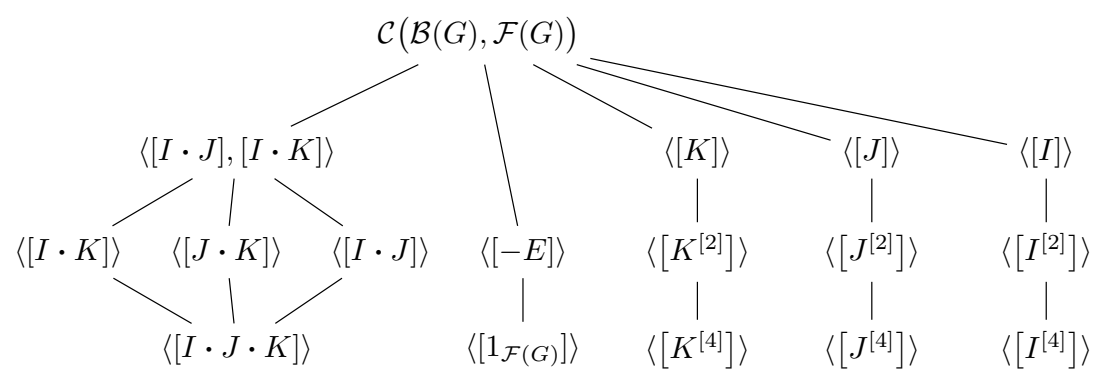

Figure 1. Subgroup Lattice of the Class Semigroup over $Q_{8}$

Example 4.2. Let $G=D_{8}=\langle a, b| a^{4}=b^{2}=1_{G}$ and $\left.b a=a^{3} b\right\rangle=\left\{1_{G}, a, a^{2}, a^{3}, b, a b, a^{2} b, a^{3} b\right\}$ be the dihedral group of order 8. Then $\mathrm{Z}(G)=G^{\prime}=\left\langle a^{2}\right\rangle=\left\{1_{G}, a^{2}\right\}$ and $G / G^{\prime} \simeq C_{2} \oplus C_{2}$. Furthermore, $\mathrm{d}(G)=4$ and $\mathrm{D}(G)=6$ by [17, Theorem 1.1].

All arguments run along the same lines as the ones given in the previous example. However, in this case, there are two elements $g, h \in G \backslash \mathbf{Z}(G)$ such that $g h=h g$. Consider the sequence $b \cdot a^{2} b \in \mathcal{F}(G)$ having $\pi\left(b \cdot a^{2} b\right)=\left\{a^{2}\right\}$, and suppose that $b \cdot a^{2} b \sim S$ for some $S \in \mathcal{F}(G)$. By Lemma 3.6 (items 1 and 5), we have $\pi(S)=\left\{a^{2}\right\}$ and hence $\langle\operatorname{supp}(S)\rangle \subset G$ is abelian subgroup containing $a^{2}$. It follows that $\langle\operatorname{supp}(S)\rangle$ is one of $\langle a\rangle,\left\langle a^{2}\right\rangle,\left\langle a^{2}, b\right\rangle$ and $\left\langle a^{2}, a b\right\rangle$.

CASE 1. $S \in \mathcal{F}\left(\left\langle a^{2}\right\rangle\right)$.

Then $S=a^{2}$ is only possible choice, but it never happen by Lemma 3.9. 3 .

CASE 2. $S \in \mathcal{F}(\langle a\rangle)$.

Then $S=a^{[2]}$ is only possible choice by Lemma 3.6 4 and Lemma 3.9 (items 1 and 2). Since $\mathcal{B}(G)$ equivalence is a congruence relation on $\mathcal{F}(G)$,

$$
b \cdot a^{2} b \sim a^{[2]} \text { implies that } b \cdot a^{2} b \cdot a \sim a^{[3]} .
$$

But $\left\{a^{3}\right\}=\pi\left(a^{[3]}\right)=\pi\left(b \cdot a^{2} b \cdot a\right)=\left\{a, a^{3}\right\}$, a contradiction.

In the case that $S \in \mathcal{F}\left(\left\langle a^{2}, a b\right\rangle\right)$, we can obtain a contradiction by the same argument of CASE 2 . It follows that $S=b \cdot a^{2} b$, and by Lemma $3.6,4$,

$$
S^{[2]} \sim b^{[2]} \text { is an idempotent element of the class semigroup } \mathcal{C}(\mathcal{B}(G), \mathcal{F}(G))
$$

with the relation $b^{[2]} \sim\left(a^{2} b\right)^{[2]} \sim S^{[2]}$.

For the sequence $T=a b \cdot a^{3} b \in \mathcal{F}(G)$, we can obtain the same result by above argument. Thus $\left[T^{[2]}\right]$ is an idempotent element of the class semigroup $\mathcal{C}(\mathcal{B}(G), \mathcal{F}(G))$ with the relation $(a b)^{[2]} \sim\left(a^{3} b\right)^{[2]} \sim T^{[2]}$. It follows that

$$
|\mathcal{C}(\mathcal{B}(G), \mathcal{F}(G))|=18 .
$$

Figure 2 presents the subgroup lattice of the class semigroup. Note that, as in the previous example, the subgroup lattice of $G$ is not preserved in the class semigroup $\mathcal{C}((\mathcal{B}(G), \mathcal{F}(G))$. Furthermore, observe that the bottom elements in the lattice are all idempotent elements of the class semigroup.

Remark 4.3. In general, the group of units $\mathcal{C}(\mathcal{B}(G), \mathcal{F}(G))^{\times}$of the class semigroup is not a direct factor. For example, let $G$ be the group described in Example 4.1. Assume to the contrary that $\mathcal{C}(\mathcal{B}(G), \mathcal{F}(G))$ has a decomposition of the form

$$
\mathcal{C}(\mathcal{B}(G), \mathcal{F}(G))=\mathcal{C}_{0} \times \mathcal{C}(\mathcal{B}(G), \mathcal{F}(G))^{\times},
$$

where $\mathcal{C}_{0}$ is a subsemigroup having 9 elements. Figure 1 . shows that there are three elements $x \in$ $\mathcal{C}(\mathcal{B}(G), \mathcal{F}(G))$ such that $x \neq x^{3}$. It follows that there exist three elements satisfying such property in the 


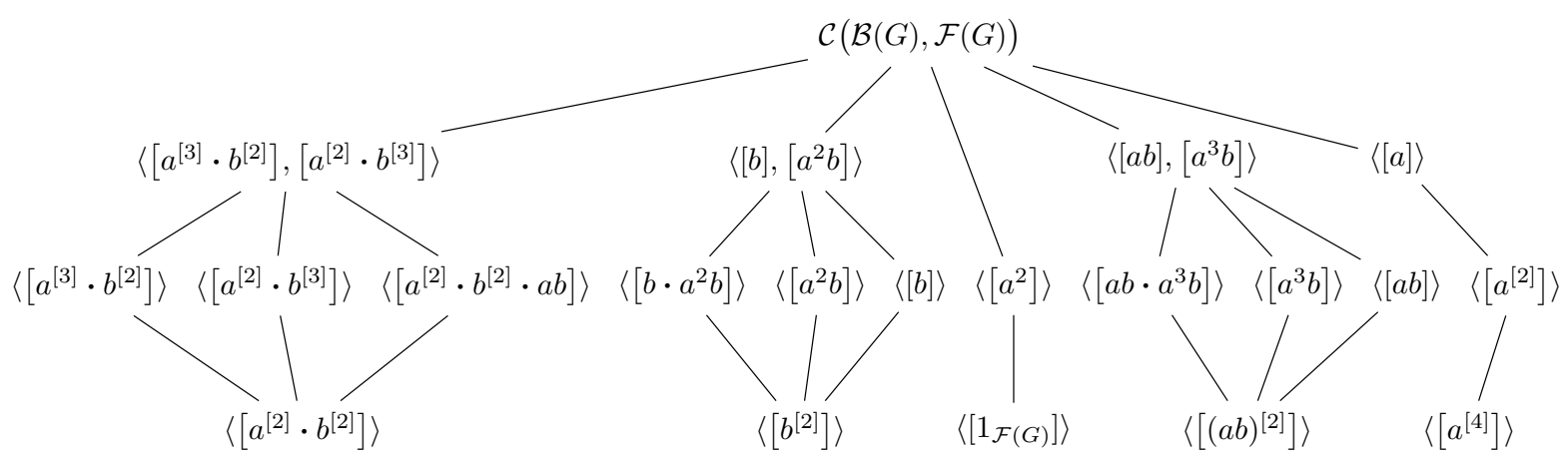

FiguRE 2. Subgroup Lattice of the Class Semigroup over $D_{8}$

decomposition. Since $\left|\mathcal{C}(\mathcal{B}(G), \mathcal{F}(G))^{\times}\right|=2, \mathcal{C}_{0}$ has at least two such elements, whence $\left|\mathcal{C}_{0}\right|>9$. Indeed, if $[I]$ and $[J]$ are in $\mathcal{C}_{0}$, then $[I \cdot J]=[I]+[J] \in \mathcal{C}_{0}$ and thus we obtain that $\mathcal{C}_{0}$ has at least 10 elements. The similar argument works for the group described in Example 4.2 .

Example 4.4. Let $G=D_{6}=\langle a, b| a^{3}=b^{2}=1_{G}$ and $\left.b a=a^{2} b\right\rangle=\left\{1_{G}, a, a^{2}, b, a b, a^{2} b\right\}$ be the dihedral group of order 6. Then $\mathrm{Z}(G)=\left\{1_{G}\right\}, G^{\prime}=\langle a\rangle=\left\{1_{G}, a, a^{2}\right\}$, and $G / G^{\prime} \simeq C_{2}$. Furthermore, $\mathrm{d}(G)=3$ and $\mathrm{D}(G)=6$ by [17, Theorem 1.1].

Let $S \in \mathcal{F}(G)$. If $|\pi(S)|=3$, then, by Theorem 3.8 , 1 , we obtain

$$
S \sim a^{[2]} \cdot b^{[2]} \quad \text { or } \quad S \sim a \cdot a^{2} \cdot b,
$$

where $\pi\left(a^{[2]} \cdot b^{[2]}\right)=\left\{1_{G}, a, a^{2}\right\} \quad$ and $\pi\left(a \cdot a^{2} \cdot b\right)=\left\{b, a b, a^{2} b\right\}$.

If $|\pi(S)|=2$, then $S$ is $\mathcal{B}(G)$-equivalent with one of the following sequences :

$$
\begin{aligned}
& a \cdot b^{[n]}, \quad a \cdot(a b)^{[n]}, \quad a \cdot\left(a^{2} b\right)^{[n]}, \quad a^{2} \cdot b^{[n]}, \quad a^{2} \cdot(a b)^{[n]}, \quad a^{2} \cdot\left(a^{2} b\right)^{[n]}, \\
& b^{[n]} \cdot a b, \quad b \cdot(a b)^{[n]}, \quad b^{[n]} \cdot a^{2} b, \quad b \cdot\left(a^{2} b\right)^{[n]}, \quad(a b)^{[n]} \cdot a^{2} b, \quad a b \cdot\left(a^{2} b\right)^{[n]},
\end{aligned}
$$

where $n \in \mathbb{N}$.

We now start with the following claims.

CLAIM.A : $\quad b^{[2]} \sim b^{[4]}, \quad(a b)^{[2]} \sim(a b)^{[4]}, \quad\left(a^{2} b\right)^{[2]} \sim\left(a^{2} b\right)^{[4]}, \quad a^{[2]} \sim a^{[5]}, \quad\left(a^{2}\right)^{[2]} \sim\left(a^{2}\right)^{[5]}$,

$$
\begin{aligned}
& \left(a^{2}\right)^{[2]} \sim a^{[4]}, \quad\left(a^{2}\right)^{[3]} \sim a^{[3]}, \quad\left(a^{2}\right)^{[4]} \sim a^{[2]}, \quad a \cdot a^{2} \sim a^{[3]}, \\
& a \cdot b \sim a^{2} \cdot b \sim a \cdot b^{[3]} \sim b^{[2]} \cdot a b \sim b^{[2]} \cdot a^{2} b, \\
& a \cdot a b \sim a^{2} \cdot a b \sim a \cdot(a b)^{[3]} \sim a b^{[2]} \cdot a^{2} b \sim a b^{[2]} \cdot b, \\
& a \cdot a^{2} b \sim a^{2} \cdot a^{2} b \sim a \cdot\left(a^{2} b\right)^{[3]} \sim\left(a^{2} b\right)^{[2]} \cdot a b \sim\left(a^{2} b\right)^{[2]} \cdot b .
\end{aligned}
$$

CLAIM.B : $\quad b \nsim b^{[3]}, \quad a b \nsim(a b)^{[3]}, \quad a^{2} b \nsim\left(a^{2} b\right)^{[3]}, \quad a \nsim a^{[4]}, \quad a^{[2]} \nsim a^{2}$,

$$
\begin{aligned}
& b \cdot a b \nsim b \cdot a^{2} b, \quad a b \cdot a^{2} b, \quad a \cdot b^{[2]}, \quad a \cdot(a b)^{[2]}, \quad a \cdot\left(a^{2} b\right)^{[2]}, \\
& b \cdot a^{2} b \nsim a b \cdot a^{2} b, \quad a \cdot b^{[2]}, \quad a \cdot(a b)^{[2]}, \quad a \cdot\left(a^{2} b\right)^{[2]}, \\
& a b \cdot a^{2} b \nsim a \cdot b^{[2]}, \quad a \cdot(a b)^{[2]}, \quad a \cdot\left(a^{2} b\right)^{[2]} .
\end{aligned}
$$

Suppose the Claims hold true. Then we obtain that

$$
|\mathcal{C}(\mathcal{B}(G), \mathcal{F}(G))|=26 \quad \text { and } \quad \mathcal{C}(\mathcal{B}(G), \mathcal{F}(G))=G_{1} \cup G_{2} \cup G_{3} \cup G_{4},
$$

where

$$
\text { - } G_{1}=\left\{[b],\left[b^{[2]}\right],\left[b^{[3]}\right],[a b],\left[(a b)^{[2]}\right],\left[(a b)^{[3]}\right],\left[a^{2} b\right],\left[\left(a^{2} b\right)^{[2]}\right],\left[\left(a^{2} b\right)^{[3]}\right],[a],\left[a^{[2]}\right],\left[a^{[3]}\right],\left[a^{[4]}\right],\left[a^{2}\right]\right\},
$$


- $G_{2}=\left\{[a \cdot b],[a \cdot a b],\left[a \cdot a^{2} b\right],\left[a \cdot b^{[2]}\right],\left[a \cdot(a b)^{[2]}\right],\left[a \cdot\left(a^{2} b\right)^{[2]}\right],[b \cdot a b],\left[b \cdot a^{2} b\right],\left[a b \cdot a^{2} b\right]\right\}$,

- $G_{3}=\left\{\left[a^{[2]} \cdot b^{[2]}\right],\left[a \cdot a^{2} \cdot b\right]\right\}$ (that is isomprphic to $\left.G / G^{\prime}\right)$, and

- $G_{4}=\left\{\left[1_{\mathcal{F}(G)}\right]\right\}$ (that is isomorphic to $\mathrm{Z}(G)$ ).

Figure 3 presents the subgroup lattice of the class semigroup. Note that, as in the previous example, the subgroup lattice of $G$ is not preserved in the class semigroup $\mathcal{C}(\mathcal{B}(G), \mathcal{F}(G))$. Furthermore, observe that the elements in the set

$$
\left\{[b],[a b],\left[a^{2} b\right],[a],\left[a^{2}\right]\right\} \cup G_{2}
$$

are not regular, whence $\mathcal{C}(\mathcal{B}(G), \mathcal{F}(G))$ is not Clifford semigroup, and the bottom elements in the lattice are all idempotent elements of the class semigroup.

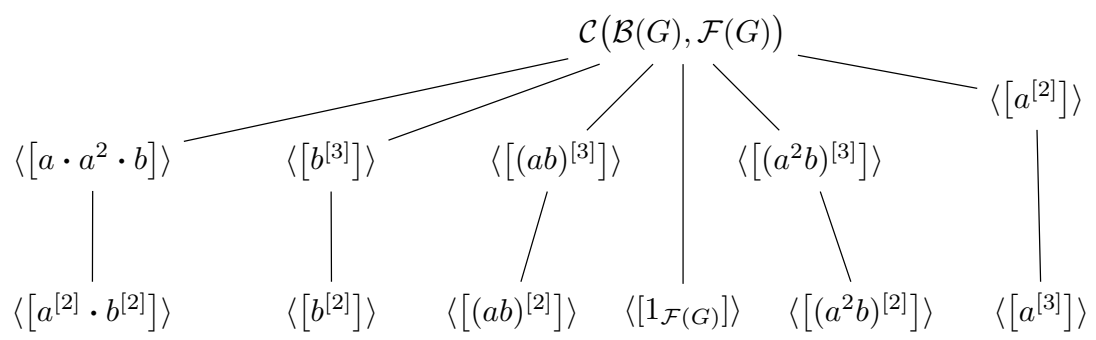

Figure 3. Subgroup Lattice of Class Semigroup over $D_{6}$

Proof of the Claim. We only show the first assertions, and others can be proved by the same way. Let $T \in \mathcal{F}(G)$. Since $b^{2}=1_{G}$, it suffices to show that if $b^{[4]} \cdot T \in \mathcal{B}(G)$, then $b^{[2]} \cdot T \in \mathcal{B}(G)$. Suppose that $b^{[4]} \cdot T \in \mathcal{B}(G)$.

CASE $1 .|\pi(T)|=3$.

Then we have

$$
T \sim a^{[2]} \cdot b^{[2]} \quad \text { or } \quad T \sim a \cdot a^{2} \cdot b .
$$

Since $b^{[4]} \cdot T \in \mathcal{B}(G)$, it follows that we have the only case $T \sim a^{[2]} \cdot b^{[2]}$. Then, since $b^{[2]} \cdot a \cdot b \cdot a \cdot b \in \mathcal{B}(G)$,

$$
b^{[2]} \cdot T \sim b^{[2]} \cdot a^{[2]} \cdot b^{[2]} \text { implies that } b^{[2]} \cdot T \in\left[b^{[2]} \cdot a^{[2]} \cdot b^{[2]}\right] \subset \mathcal{B}(G) .
$$

CASE 2. $|\pi(T)|=2$.

Then $T$ is $\mathcal{B}(G)$-equivalent with one of the sequence described in (4.1). Since $b^{[4]} \cdot T \in \mathcal{B}(G)$, the possible choice of $T$ under $\mathcal{B}(G)$-equivalence is one the following sequences:

$$
\begin{array}{cccc}
a \cdot(a b)^{[\text {even] }}, & a \cdot\left(a^{2} b\right)^{[\text {even }]}, & a^{2} \cdot(a b)^{[\text {even }]}, & a^{2} \cdot\left(a^{2} b\right)^{[\text {even] }}, \\
b \cdot(a b)^{[\text {odd } \geq 3]}, & b \cdot\left(a^{2} b\right)^{[\text {odd } \geq 3]}, & (a b)^{[\text {odd }]} \cdot a^{2} b, & a b \cdot\left(a^{2} b\right)^{[\text {odd }]} .
\end{array}
$$

Then we obtain the following simple calculation, and it can cover all other cases:

$$
\begin{gathered}
a b \cdot b \cdot a b \cdot b \cdot a, \quad a^{2} b \cdot b \cdot a^{2} b \cdot a \cdot b, \quad a b \cdot b \cdot a b \cdot a^{2} \cdot b, \quad a^{2} b \cdot b \cdot a^{2} b \cdot b \cdot a^{2}, \\
a b \cdot b \cdot a b \cdot b \cdot a b \cdot b, \quad a^{2} b \cdot b \cdot a^{2} b \cdot b \cdot a^{2} b \cdot b, \quad a b \cdot b \cdot a^{2} b \cdot b,
\end{gathered}
$$

which are all product-one sequences, and thus it follows that $b^{[2]} \cdot T \in \mathcal{B}(G)$.

CASE 3. $|\pi(T)|=1$.

Then $\langle\operatorname{supp}(T)\rangle$ is abelian subgroup by Lemma 3.6.5. If $\langle\operatorname{supp}(T)\rangle=\left\langle 1_{G}\right\rangle$, then we are done because $T \in \mathcal{B}(G)$. 
If $\langle\operatorname{supp}(T)\rangle=\langle b\rangle$, then $T=b^{[n]}$ for some $n \in \mathbb{N}$ by Lemma 3.6 4. Since $b^{[4]} \cdot T \in \mathcal{B}(G)$, it follows that $n$ should be even number, and hence we are done.

By symmetry, we can obtain the same result whenever $\langle\operatorname{supp}(T)\rangle=\langle a b\rangle$ or $\left\langle a^{2} b\right\rangle$.

Suppose now that $\langle\operatorname{supp}(T)\rangle=\langle a\rangle$. Then $T=a^{\left[n_{1}\right]} \cdot\left(a^{2}\right)^{\left[n_{2}\right]}$ for some $n_{1}, n_{2} \in \mathbb{N}_{0}$.

i) $n_{1}=0$.

In this case, $b^{[4]} \cdot\left(a^{2}\right)^{\left[n_{2}\right]} \in \mathcal{B}(G)$ implies that $n_{2} \geq 2$. It follows that $b^{[2]} \cdot\left(a^{2}\right)^{\left[n_{2}\right]} \in \mathcal{B}(G)$.

ii) $n_{2}=0$.

In this case, $b^{[4]} \cdot a^{\left[n_{1}\right]} \in \mathcal{B}(G)$ implies that $n_{1} \geq 2$. It follows that $b^{[2]} \cdot a^{\left[n_{1}\right]} \in \mathcal{B}(G)$.

iii) $n_{1} \geq 1$ and $n_{2} \geq 1$.

To avoid the trivial case, we may assume that $n_{1} \neq n_{2}$.

If $\left|n_{1}-n_{2}\right|=1$, then, since any choice of $n_{1}, n_{2}$ can be reduced to the case

$$
n_{1}=1, n_{2}=2 \quad \text { or } \quad n_{1}=2, n_{2}=1,
$$

it suffices to verify only such two cases, and we have the followings:

$$
\begin{array}{r}
a \cdot b \cdot a^{2} \cdot a^{2} \cdot b^{[3]} \in \mathcal{B}(G) \quad \text { implies that } \quad a \cdot b \cdot a^{2} \cdot a^{2} \cdot b \in \mathcal{B}(G), \\
a \cdot a \cdot b \cdot a^{2} \cdot b^{[3]} \in \mathcal{B}(G) \quad \text { implies that } \quad a \cdot a \cdot b \cdot a^{2} \cdot b \in \mathcal{B}(G) .
\end{array}
$$

If $\left|n_{1}-n_{2}\right| \geq 2$, then it can be reduced to the case $n_{1}=0$ or $n_{2}=0$.

Therefore, in any cases, we can obtain $b^{[2]} \cdot T \in \mathcal{B}(G)$, and it follows that $b^{[2]} \sim b^{[4]}$. Moreover, $b \nsim b^{[3]}$ because $b^{[3]} \cdot(a b)^{[3]} \in \mathcal{B}(G)$, but $b \cdot(a b)^{[3]} \notin \mathcal{B}(G)$. Hence

$$
[b], \quad\left[b^{[2]}\right], \quad\left[b^{[3]}\right]
$$

are distinct $\mathcal{B}(G)$-equivalence classes in the class semigroup $\mathcal{C}(\mathcal{B}(G), \mathcal{F}(G))$.

\section{Arithmetic Properties of the monoid of product-one sequences}

The goal of this section is to study the arithmetic of the monoid $\mathcal{B}(G)$ of product-one sequences. To do so we briefly recall some arithmetical concepts (details can be found in [18]).

Let $H$ be an atomic monoid and $a, b \in H$. If $a=u_{1} \cdot \ldots \cdot u_{k}$, where $k \in \mathbb{N}$ and $u_{1}, \ldots, u_{k} \in \mathcal{A}(H)$, then $k$ is called the length of the factorization and $\mathrm{L}_{H}(a)=\mathrm{L}(a)=\{k \in \mathbb{N} \mid a$ has a factorization of length $k\} \subset \mathbb{N}$ is the set of lengths of $a$. As usual we set $\mathrm{L}(a)=\{0\}$ if $a \in H^{\times}$, and then

$$
\mathcal{L}(H)=\{\mathrm{L}(a) \mid a \in H\}
$$

denotes the system of sets of lengths of $H$. Let $k \in \mathbb{N}$ and suppose that $H \neq H^{\times}$. Then

$$
\mathcal{U}_{k}(H)=\bigcup_{k \in L, L \in \mathcal{L}(H)} L \subset \mathbb{N}
$$

denotes the union of sets of lengths containing $k$, and we set

$$
\rho_{k}(H)=\sup \mathcal{U}_{k}(H) .
$$

If $L=\left\{m_{1}, \ldots, m_{k}\right\} \subset \mathbb{Z}$ is a finite subset of the integers, where $k \in \mathbb{N}$ and $m_{1}<\ldots<m_{k}$, then $\Delta(L)=\left\{m_{i}-m_{i-1} \mid i \in[2, k]\right\} \subset \mathbb{N}$ is the set of distances of $L$. If all sets of lengths are finite, then

$$
\Delta(H)=\bigcup_{L \in \mathcal{L}(H)} \Delta(L)
$$

is the set of distances of $H$. It is easy to check that $\min \Delta(H)=\operatorname{gcd} \Delta(H)$. If $\theta: H \rightarrow B$ is a transfer homomorphism between atomic monoids having finite sets of lengths, then ([18, Proposition 3.2.3])

$$
\mathcal{L}(H)=\mathcal{L}(B) \text { whence } \Delta(H)=\Delta(B) \text { and } \mathcal{U}_{k}(H)=\mathcal{U}_{k}(B) \text { for all } k \in \mathbb{N} \text {. }
$$


It is well-known that if $H$ is finitely generated, then then all unions $\mathcal{U}_{k}(H)$ of sets of lengths (in particular, all sets of lengths) and the set of distances $\Delta(H)$ are finite ([18, Theorem 3.1.4]).

We will study the system of sets of lengths and all further mentioned invariants for the monoid $\mathcal{B}(G)$. As usual, we set

$$
\mathcal{L}(G)=\mathcal{L}(\mathcal{B}(G)), \Delta(G)=\Delta(\mathcal{B}(G)), \mathcal{U}_{k}(G)=\mathcal{U}_{k}(\mathcal{B}(G))
$$

and $\rho_{k}(G)=\rho_{k}(\mathcal{B}(G))$ for all $k \in \mathbb{N}$. Since $\mathcal{B}(G)$ is finitely generated by Lemma 3.2, all these invariants are finite. The significance of the monoid $\mathcal{B}(G)$ for abelian groups stems from its relevance for general Krull monoids. Indeed, if $H$ is a commutative Krull monoid with class group $G$ such that every class contains a prime divisor, then there is a transfer homomorphism $\theta: H \rightarrow \mathcal{B}(G)$ and hence $\mathcal{L}(H)=\mathcal{L}(G)$. This transfer process from monoids of zero-sum sequences to more general monoids carries over to transfer Krull monoids ([16]).

We study the structure of $\mathcal{U}_{k}(G)$ and of $\Delta(G)$ for general finite groups $G$ and we will derive canonical bounds for their size. Most results are known in the abelian case partly with different bounds (for a recent survey on the abelian case we refer to [32]). However, if $G$ is non-abelian, then $\mathcal{B}(G)$ is not a transfer Krull monoid by Proposition 3.4. Thus the present results cannot be derived from the abelian setting. If $|G| \leq 2$, then $\mathcal{B}(G)$ is factorial, $\mathcal{L}(G)=\left\{\{k\} \mid k \in \mathbb{N}_{0}\right\}, \Delta(G)=\emptyset$, and $\mathcal{U}_{k}(G)=\{k\}$ for all $k \in \mathbb{N}$. To avoid annoying case distinctions we exclude this trivial case.

\section{Throughout this section, let $G$ be a finite group with $|G| \geq 3$.}

Although the forthcoming proofs parallel the proofs given in the commutative setting there is a main difference. It stems from the fact that, in the non-abelian case, the embedding $\mathcal{B}(G) \hookrightarrow \mathcal{F}(G)$ is not a divisor homomorphism. Thus there exist $U, V \in \mathcal{B}(G)$ such that $U$ divides $V$ in $\mathcal{F}(G)$, but not in $\mathcal{B}(G)$. Moreover, $U$ and $V$ can be atoms (e.g., if $G$ is the quaternion group, as discussed in Examples 4.1, then $U=I^{[4]} \in \mathcal{A}(G)$ and $V=I^{[4]} \cdot J^{[2]} \in \mathcal{A}(G)$ have this property).

Lemma 5.1. Let $k, \ell \in \mathbb{N}$ with $k<\ell$ and $U_{1}, \ldots, U_{k}, V_{1}, \ldots, V_{\ell} \in \mathcal{A}(G)$ such that $U_{1} \cdot \ldots \cdot U_{k}=V_{1} \cdot \ldots \cdot V_{\ell}$. There exist $\mu \in[1, k], \lambda, \lambda^{\prime} \in[1, \ell]$ with $\lambda \neq \lambda^{\prime}$, and $g_{1}, g_{2} \in G$ such that $U_{\mu}=g_{1} \cdot g_{2} \cdot \ldots \cdot g_{m}$ with $m \geq 2$, $1_{G}=g_{1} \ldots g_{m}, g_{1} \mid V_{\lambda}$, and $g_{2} \mid V_{\lambda^{\prime}}$ in $\mathcal{F}(G)$.

Proof. Assume to the contrary that the assertion does not hold. Since $1_{G}$ is a prime element of $\mathcal{B}(G)$, we may suppose without restriction that all $U_{i}$ and $V_{j}$ have length at least two. We set $U_{1}=g_{1} \cdot g_{2} \cdot \ldots \cdot g_{m}$ where $m \geq 2$ and $1_{G}=g_{1} \ldots g_{m}$. Then $g_{1} \cdot g_{2}$ divides $V_{\lambda}$ for some $\lambda \in[1, \ell]$, say $\lambda=1$. Then we consider $g_{2} \cdot g_{3}$. Since the assertion does not hold, it follows that $g_{1} \cdot g_{2} \cdot g_{3}$ divides $V_{1}$. Proceeding recursively we obtain that $U_{1} \mid V_{1}$, say $V_{1}=U_{1} \cdot V_{1}^{\prime}$ with $V_{1}^{\prime} \in \mathcal{F}(G)$. Thus we obtain the equation

$$
U_{2} \cdot \ldots \cdot U_{k}=V_{1}^{\prime} \cdot V_{2} \cdot \ldots \cdot V_{\ell} \text {. }
$$

Proceeding in this way we end up with an equation of the form

$$
1_{\mathcal{F}(G)}=S_{1} \cdot \ldots \cdot S_{\eta} \cdot V_{\eta+1} \cdot \ldots \cdot V_{\ell},
$$

where $\eta \leq k$ and $S_{1}, \ldots, S_{\eta} \in \mathcal{F}(G)$, a contradiction to $k<\ell$.

We consider the following property:

P. If $U=g_{1} \cdot \ldots \cdot g_{\ell} \in \mathcal{A}(G)$ and $g_{1}=h_{1} h_{2}$ with $h_{1}, h_{2} \in G$, then $U^{\prime}=h_{1} \cdot h_{2} \cdot g_{2} \cdot \ldots \cdot g_{\ell}$ is either an atom or a product of two atoms at most.

Remark 5.2. Of course, every abelian group satisfies Property $\mathbf{P}$ and the same is true for some nonabelian groups such as the quaternion group. However, for every $n \geq 9$, the dihedral group $D_{2 n}$ does not have Property $\mathbf{P}$ as the following example shows. 
Example 5.3. 1. Let $G=Q_{8}$ be the quaternion group as discussed in Example 4.1. Then $\mathrm{D}(G)=6$, and clearly any atom having length at most 4 satisfies Property $\mathbf{P}$. Any atom $U$ having the length 6 has the form

$$
U=g_{1}^{[4]} \cdot g_{2}^{[2]}, \quad \text { where } g_{1}, g_{2} \in\{I, J, K,-I,-J,-K\} \text { with } g_{2} \neq \pm g_{1},
$$

and any atom $V$ having length 5 is one of the following three types :

- $V=g_{1}^{[3]} \cdot g_{2} \cdot g_{3}, \quad$ where $g_{1}, g_{2}, g_{3} \in\{I, J, K,-I,-J,-K\}$ with $g_{2} \neq g_{3}$ and $g_{2}, g_{3} \neq \pm g_{1}$.

- $V=g_{1}^{[2]} \cdot g_{2}^{[2]} \cdot(-E)$, where $g_{1}, g_{2} \in\{I, J, K,-I,-J,-K\}$ with $g_{2} \neq \pm g_{1}$.

- $V=g_{1} \cdot\left(-g_{1}\right) \cdot g_{2} \cdot\left(-g_{2}\right) \cdot(-E)$, where $g_{1}, g_{2} \in\{I, J, K\}$ with $g_{1} \neq g_{2}$.

It is easy to check that $G$ satisfies Property $\mathbf{P}$.

2. Let $G=D_{2 n}=\langle a, b| a^{n}=b^{2}=1_{G}$ and $\left.b a=a^{-1} b\right\rangle$ be the dihedral group, where $n \geq 9$. Consider the following sequences:

$$
U=b^{[2]} \cdot(b a)^{[3]} \cdot a^{2} \cdot b a^{5} \quad \text { and } \quad U^{\prime}=b^{[2]} \cdot(b a)^{[3]} \cdot b a^{2} \cdot b a^{4} \cdot b a^{5} .
$$

Then $U^{\prime}$ is a product of $b^{[2]},(b a)^{[2]}$, and $b a^{2} \cdot b a \cdot b a^{4} \cdot b a^{5}$, which are atoms. Now we need to verify that $U$ is an atom. Assume to the contrary that $U$ is not atom, say $U=W_{1} \cdot W_{2}$ with $W_{1}, W_{2} \in \mathcal{B}(G)$, and let $W_{1}$ be an atom with $a^{2} \mid W_{1}$. Write $W_{1}=g_{1} \cdot g_{2} \cdot \ldots \cdot g_{\ell}$ with $g_{1}=a^{2}$ and $g_{1} g_{2} \ldots g_{\ell}=1_{G}$. Then $\ell=3$ or $\ell=5$. If $\ell=3$, then $g_{2} g_{3}=a^{-2}$ and $g_{2}, g_{3} \in\left\{b, b a, b a^{5}\right\}$. Since $n \geq 9$, this is impossible. If $\ell=5$, then $W_{2}=b^{[2]}$ or $W_{2}=(b a)^{[2]}$ which implies that $W_{1}=(b a)^{[3]} \cdot a^{2} \cdot b a^{5}$ or $W_{1}=b^{[2]} \cdot b a \cdot a^{2} \cdot b a^{5}$. It is easy to check that $W_{1}$ is not an atom. Thus $G$ does not have Property $\mathbf{P}$.

Lemma 5.4. Suppose that $G$ satisfies Property $\mathbf{P}$. Then for every $S \in \mathcal{B}(G)$ with $\max \Delta(\mathrm{L}(S)) \geq 2$, there exists $T \in \mathcal{B}(G)$ such that $|T|<|S|$ and $\max \Delta(\mathrm{L}(T)) \geq \max \Delta(\mathrm{L}(S))-1$.

Proof. Let $S \in \mathcal{B}(G)$ and $d=\max \Delta(\mathrm{L}(S)) \geq 2$. Then there are $k, \ell \in \mathbb{N}$ and $U_{1}, \ldots, U_{k}, V_{1}, \ldots, V_{\ell} \in$ $\mathcal{A}(G)$ such that

$$
S=U_{1} \cdot \ldots \cdot U_{k}=V_{1} \cdot \ldots \cdot V_{\ell}
$$

with $\ell-k=d$ and $U_{1} \cdot \ldots \cdot U_{k}$ has no factorization of length in $[k+1, \ell-1]$. Since $1_{G} \in \mathcal{B}(G)$ is a prime element, we may assume that $1_{G}$ does not divide $S$, and thus all $U_{i}$ and $V_{j}$ have length at least 2 .

By Lemma [5.1, we may assume that there are $g_{1}, g_{2} \in G$ such that

$$
g_{1} \cdot g_{2}\left|U_{1}, \quad g_{1}\right| V_{1} \text { and } g_{2} \mid V_{2} \text { in } \mathcal{F}(G) \text {. }
$$

Let $U_{1}=g_{1} \cdot g_{2} \cdot U_{1}^{\prime}, V_{1}=g_{1} \cdot V_{1}^{\prime}$ and $V_{2}=g_{2} \cdot V_{2}^{\prime}$ with $U_{1}^{\prime}, V_{1}^{\prime}, V_{2}^{\prime} \in \mathcal{F}(G)$. Then we set $g_{0}=g_{1} g_{2} \in G$, and consider

$$
U_{1}^{\prime \prime}=g_{0} \cdot U_{1}^{\prime} \text { and } V_{1}^{\prime \prime}=g_{0} \cdot V_{1}^{\prime} \cdot V_{2}^{\prime} \text {. }
$$

Clearly, $U_{1}^{\prime \prime} \in \mathcal{A}(G)$. Since $V_{1}$ gives rise to a product-one equation with $g_{1}$ being the finial element and $V_{2}$ gives rise to a product-one equation with $g_{2}$ being the first element, it follows that $V_{1}^{\prime \prime} \in \mathcal{B}(G)$. We obtain that

$$
U_{1}^{\prime \prime} \cdot U_{2} \cdot \ldots \cdot U_{k}=V_{1}^{\prime \prime} \cdot V_{3} \cdot \ldots \cdot V_{\ell}
$$

We set $T=U_{1}^{\prime \prime} \cdot U_{2} \cdot \ldots \cdot U_{k}$ and observe that $|T|<|S|$. If $T=W_{1} \cdot \ldots \cdot W_{t}$ with $W_{1}, \ldots, W_{t} \in \mathcal{A}(G)$ and $g_{0} \mid W_{1}$ in $\mathcal{F}(G)$, then $W_{1}=g_{0} \cdot W_{1}^{\prime}$ and $W_{1}^{\prime \prime}=g_{1} \cdot g_{2} \cdot W_{1}^{\prime}$ is either atom or product of precisely two atoms. Thus $S=U_{1} \cdot \ldots \cdot U_{k}$ has a factorization of length $t$ or $t+1$. It follows that $T$ has no factorization of length in $[k+1, \ell-2]$, and thus we obtain

$$
\max \Delta(\mathrm{L}(T)) \geq \ell-1-k=d-1=\max \Delta(\mathrm{L}(S))-1
$$

The next result shows that the set of distances and all unions of sets of lengths of $\mathcal{B}(G)$ are finite intervals. This is far from being true in general. Indeed, for every finite set $\Delta \subset \mathbb{N}$ with $\min \Delta=\operatorname{gcd} \Delta$ there is a finitely generated Krull monoid $H$ such that $\Delta(H)=\Delta([22])$. 


\section{Theorem 5.5.}

1. $\mathcal{U}_{k}(G)$ is a finite interval for all $k \in \mathbb{N}$.

2. If $G$ satisfies Property $\mathbf{P}$, then $\Delta(G)$ is a finite interval with $\min \Delta(G)=1$.

Proof. 1. Let $k \in \mathbb{N}$. We set $\lambda_{k}(G)=\min \mathcal{U}_{k}(G)$. Then $\mathcal{U}_{k}(G) \subset\left[\lambda_{k}(G), \rho_{k}(G)\right]$, and we have to show equality. Note that it is sufficient to prove that $\left[k, \rho_{k}(G)\right] \subset \mathcal{U}_{k}(G)$. Indeed, suppose that this is done, and let $\ell \in\left[\lambda_{k}(G), k\right]$. Then $\ell \leq k \leq \rho_{\ell}(G)$, hence $k \in \mathcal{U}_{\ell}(G)$ and consequently $\ell \in \mathcal{U}_{k}(G)$. It follows that $\left[\lambda_{k}(G), \rho_{k}(G)\right]=\mathcal{U}_{k}(G)$.

To prove the assertion, let $\ell \in\left[k, \rho_{k}(G)\right]$ be minimal such that $\left[\ell, \rho_{k}(G)\right] \subset \mathcal{U}_{k}(G)$. Assume to the contrary that $\ell>k$. We set $\Omega=\{A \in \mathcal{B}(G) \mid\{k, j\} \subset \mathrm{L}(A)$ for some $j \geq \ell\}$ and choose $B \in \Omega$ such that $|B|$ is minimal. Then

$$
B=U_{1} \cdot \ldots \cdot U_{k}=V_{1} \cdot \ldots \cdot V_{j}, \quad \text { where } U_{1}, \ldots, U_{k}, V_{1}, \ldots, V_{j} \in \mathcal{A}(G),
$$

and by Lemma 5.1 we may assume that

$$
U_{k}=g_{1} \cdot g_{2} \cdot U \text { with } g_{1} \mid V_{j-1} \text { and } g_{2} \mid V_{j},
$$

where $U=g_{3} \cdot \ldots \cdot g_{t}$ and $g_{1} \ldots g_{t}=1_{G}$. We set $g_{0}=g_{1} g_{2} \in G$ and obtain that $U_{k}^{\prime}=g_{0} \cdot U \in \mathcal{A}(G)$. Write

$$
V_{j-1}=h_{1} \cdot \ldots \cdot h_{n} \cdot g_{1} \text { and } V_{j}=g_{2} \cdot s_{1} \cdot \ldots \cdot s_{m}
$$

with $h_{1} \ldots h_{n} g_{1}=1_{G}$ and $g_{2} s_{1} \ldots s_{m}=1_{G}$. Let $V_{j-1}^{\prime}=g_{0} \cdot V \in \mathcal{B}(G)$, where $V=h_{1} \cdot \ldots \cdot h_{n} \cdot s_{1} \cdot \ldots \cdot s_{m} \in$ $\mathcal{F}(G)$, and consider

Then $\left|B^{\prime}\right|<|B|$, and

$$
B^{\prime}=U_{1} \cdot \ldots \cdot U_{k-1} \cdot U_{k}^{\prime}
$$

$$
B^{\prime}=U_{1} \cdot \ldots \cdot U_{k-1} \cdot U_{k}^{\prime}=V_{1} \cdot \ldots \cdot V_{j-2} \cdot W_{1} \cdot \ldots \cdot W_{t}
$$

where $V_{j-1}^{\prime}=W_{1} \cdot \ldots \cdot W_{t}$ with $W_{1}, \ldots, W_{t} \in \mathcal{A}(G)$. By the minimality of $|B|$, we have $(j-2)+t<\ell \leq j$. Hence $t=1$ and $j=\ell$. Thus $\ell-1 \in \mathcal{U}_{k}(G)$, a contradiction to the minimality of $\ell$. Therefore $\ell=k$ and hence $\left[k, \rho_{k}(G)\right] \subset \mathcal{U}_{k}(G)$.

2. Since $\Delta(G)$ is finite as mentioned before, we can take $S_{0} \in \mathcal{B}(G)$ with minimal length such that $\max \Delta\left(\mathrm{L}\left(S_{0}\right)\right)=\max \Delta(G)$. By Lemma 5.4, we can find $S_{1} \in \mathcal{B}(G)$ with minimal length such that $\left|S_{1}\right|<\left|S_{0}\right|$ and $\max \Delta\left(\mathrm{L}\left(S_{1}\right)\right) \geq \max \Delta(G)-1$. By the minimality of $S_{0}$,

$$
\left|S_{1}\right|<\left|S_{0}\right| \quad \text { implies } \max \Delta\left(\mathrm{L}\left(S_{1}\right)\right)<\max \Delta(G) \text {. }
$$

It follows that $\max \Delta(G)-1=\max \Delta\left(\mathrm{L}\left(S_{1}\right)\right) \in \Delta(G)$. Again Lemma 5.4 implies that we can find $S_{2} \in \mathcal{B}(G)$ with minimal length such that $\left|S_{2}\right|<\left|S_{1}\right|$ and $\max \Delta\left(\mathrm{L}\left(S_{2}\right)\right) \geq \max \Delta(G)-2$. By the minimality of $S_{1}$,

$$
\left|S_{2}\right|<\left|S_{1}\right| \quad \text { implies } \quad \max \Delta\left(\mathrm{L}\left(S_{2}\right)\right)<\max \Delta(G)-1 .
$$

It follows that $\max \Delta(G)-2=\max \Delta\left(\mathrm{L}\left(S_{2}\right)\right) \in \Delta(G)$. Continuing this process, we can obtain $S_{n} \in \mathcal{B}(G)$ such that

$$
1=\max \Delta(G)-n=\max \Delta\left(\mathrm{L}\left(S_{n}\right)\right) \in \Delta(G) .
$$

Thus $\Delta(G)=[1, \max \Delta(G)]$ is an interval.

Next we study the maxima of the sets $\mathcal{U}_{k}(G)$. Recall that we have defined $\rho_{k}(G)=\max \mathcal{U}_{k}(G)$ for all $k \in \mathbb{N}$. Even in case of abelian groups, precise values of $\rho_{2 k+1}(G)$ are unknown in general ([11]).

Proposition 5.6. Let $k \in \mathbb{N}$.

1. $\rho_{k}(G) \leq \frac{k \mathrm{D}(G)}{2}$ for all $k \in \mathbb{N}$.

2. $\rho_{2 k}(G)=k \mathrm{D}(G)$ for all $k \in \mathbb{N}$.

In particular, $1+k \mathrm{D}(G) \leq \rho_{2 k+1}(G) \leq k \mathrm{D}(G)+\frac{\mathrm{D}(G)}{2}$ for all $k \in \mathbb{N}$. 
Proof. 1. Let $k \in \mathbb{N}$. Let $A \in \mathcal{B}(G)$ with $k \in \mathrm{L}(A)$. We have to show that $\max \mathrm{L}(A) \leq \frac{k \mathrm{D}(G)}{2}$. There are $U_{1}, \ldots, U_{k} \in \mathcal{A}(G)$ such that

$$
A=U_{1} \cdot \ldots \cdot U_{k} .
$$

Since $1_{G} \in \mathcal{B}(G)$ is a prime element, we may assume that $1_{G}$ does not divide $A$, and hence any atom dividing $A$ has length at least 2. If $A=V_{1} \cdot \ldots \cdot V_{\ell}$ for $V_{1}, \ldots, V_{\ell} \in \mathcal{A}(G)$, then we obtain

$$
2 \ell \leq \sum_{i=1}^{\ell}\left|V_{i}\right|=|A|=\sum_{j=1}^{k}\left|U_{j}\right| \leq k \mathrm{D}(G) .
$$

It follows that $\ell \leq \frac{k \mathrm{D}(G)}{2}$, and thus $\max \mathrm{L}(A) \leq \frac{k \mathrm{D}(G)}{2}$.

2. Let $k \in \mathbb{N}$. Then, by 1 ., we have $\rho_{2 k}(G) \leq k \mathrm{D}(G)$. Now let $U=g_{1} \cdot \ldots \cdot g_{\ell} \in \mathcal{A}(G)$ with $\ell=|U|=\mathrm{D}(G)$, where $g_{1}, \ldots, g_{\ell} \in G$. Consider the sequence

$$
V=g_{1}^{-1} \cdot \ldots \cdot g_{\ell}^{-1} \in \mathcal{A}(G) .
$$

Then $k \mathrm{D}(G) \in \mathrm{L}\left((U \cdot V)^{[k]}\right)$. Since $2 k \in \mathrm{L}\left((U \cdot V)^{[k]}\right)$, we obtain $\rho_{2 k}(G) \geq k \mathrm{D}(G)$, and hence $\rho_{2 k}(G)=$ $k \mathrm{D}(G)$.

It remains to prove the "In particular" statement. For all $i, j \in \mathbb{N}$, we have $\mathcal{U}_{i}(G)+\mathcal{U}_{j}(G) \subset \mathcal{U}_{i+j}(G)$ whence $\rho_{i}(G)+\rho_{j}(G) \leq \rho_{i+j}(G)$. Thus the "In particular" statement follows from 1. and 2 .

Our next goal is to study the maximum of the set of distances $\Delta(G)$. If $G$ is abelian, then precise values for $\max \Delta(G)$ are known only for very special classes of groups including cyclic groups ([23]). For general groups $G$ we study $\max \Delta(G)$ via a further invariant (introduced in Definition 5.7). The main result is Proposition 5.12.

Definition 5.7. Let $H$ be an atomic monoid.

1. For $b \in H$, let $\omega(H, b)$ denote the smallest $N \in \mathbb{N}_{0} \cup\{\infty\}$ with the following property:

For all $n \in \mathbb{N}$ and $a_{1}, \ldots, a_{n} \in H$, if $b \mid a_{1} \ldots a_{n}$, then there exists a subset $\Omega \subset[1, n]$ such that

$$
|\Omega| \leq N \text { and } b \mid \prod_{\nu \in \Omega} a_{\nu} \text {. }
$$

2. We set

$$
\omega(H)=\sup \{\omega(H, u) \mid u \in \mathcal{A}(H)\} \in \mathbb{N}_{0} \cup\{\infty\} .
$$

If $H$ is an atomic monoid with $\Delta(H) \neq \emptyset$, then $2+\max \Delta(H) \leq \omega(H)$ (see [18, Theorem 1.6.3] and [20, Proposition 3.6]). As usual we set $\omega(G)=\omega(\mathcal{B}(G))$ and observe that $2+\max \Delta(G) \leq \omega(G)$. If $G$ is a cyclic group or an elementary 2-group, then $2+\max \Delta(G)=\omega(G)=\mathrm{D}(G)$.

Lemma 5.8. We have $\mathrm{D}(G) \leq \omega(G)$, and equality holds if $G$ is abelian.

Proof. Let $U=g_{1} \cdot \ldots \cdot g_{\ell} \in \mathcal{A}(G)$ with $\ell=|U|=\mathrm{D}(G)$. Put $V_{i}=g_{i} \cdot g_{i}^{-1} \in \mathcal{A}(G)$ for all $i \in[1, \ell]$. Then we obtain

$$
U \mid V_{1} \cdot \ldots \cdot V_{\ell} \text { in } \mathcal{B}(G) .
$$

Assume to the contrary that $U$ divides a proper subproduct in $\mathcal{B}(G)$, say $U \mid V_{1} \cdot \ldots \cdot V_{k}$ for some $k \in[1, \ell-1]$. Then $2 k \geq \ell$. If $2 k=\ell$, then $U=V_{1} \cdot \ldots \cdot V_{k}$ is not atom in $\mathcal{B}(G)$. If $2 k>\ell$, then $\ell-k<k$, and, for each $j=[1, \ell-k]$, we may assume that $g_{k+j}=g_{j}^{-1}$. Hence we have

$$
U=\left(g_{1} \cdot g_{k+1}\right) \cdot \ldots \cdot\left(g_{\ell-k} \cdot g_{\ell}\right) \cdot\left(g_{\ell-k+1} \cdot \ldots \cdot g_{k}\right),
$$

and each terms is in $\mathcal{B}(G)$. It follows that $U$ is not atom in $\mathcal{B}(G)$, a contradiction. Thus $U$ cannot divide a proper subproduct, and hence $\mathrm{D}(G) \leq \omega(G)$.

Suppose that $G$ is abelian. It remains to show that $\omega(G) \leq \mathrm{D}(G)$. Let $U \in \mathcal{A}(G)$. If $U \mid V_{1} \cdot \ldots \cdot V_{k}$ for $V_{1}, \ldots, V_{k} \in \mathcal{B}(G)$, then there is a subproduct of at most $|U|$ factors such that $U$ divides this subproduct in $\mathcal{F}(G)$. Since $\mathcal{B}(G) \hookrightarrow \mathcal{F}(G)$ is a divisor homomorphism, this divisibility holds in $\mathcal{B}(G)$. Hence $\omega(G, U) \leq$ $|U| \leq \mathrm{D}(G)$, and it follows that $\omega(G) \leq \mathrm{D}(G)$. 
Next we define Davenport constants of additive commutative semigroups (as studied in [35, 1, 34]) and will apply these concepts to the class semigroup of $\mathcal{B}(G)$. Recall that all our semigroups have an identity element (a zero-element in the additive case) and we use the convention that an empty sum equals the zero-element.

Definition 5.9. Let $\mathcal{C}$ be an additive commutative semigroup.

1. We denote by $\mathrm{d}(\mathcal{C})$ the smallest $d \in \mathbb{N}_{0} \cup\{\infty\}$ with the following property :

For all $n \in \mathbb{N}$ and $c_{1}, \ldots, c_{n} \in \mathcal{C}$, there exists $\Omega \subset[1, n]$ such that $|\Omega| \leq d$ and $\sum_{\nu=1}^{n} c_{\nu}=\sum_{\nu \in \Omega} c_{\nu}$.

2. We denote by $D(\mathcal{C})$ the smallest $\ell \in \mathbb{N} \cup\{\infty\}$ with the following property :

For all $n \in \mathbb{N}$ with $n \geq \ell$ and $c_{1}, \ldots, c_{n} \in \mathcal{C}$, there exists $\Omega \subsetneq[1, n]$ such that $\sum_{\nu=1}^{n} c_{\nu}=\sum_{\nu \in \Omega} c_{\nu}$.

If $G$ is a finite abelian group, then of course the definitions for $\mathrm{D}(G)$ and $\mathrm{d}(G)$ given in Definition 3.1 and in Definition 5.9 coincide.

Lemma 5.10. If $\mathcal{C}$ is a finite commutative semigroup, then $\mathrm{D}(\mathcal{C})=\mathrm{d}(\mathcal{C})+1 \leq|\mathcal{C}|$.

Proof. The equality $\mathrm{D}(\mathcal{C})=\mathrm{d}(\mathcal{C})+1$ is verified in [1, Proposition 1.2]. Since we could not find a reference that $|\mathcal{C}|$ is an upper bound, we provide the short argument. To show that $\mathrm{D}(\mathcal{C}) \leq|\mathcal{C}|$, it suffices to verify that $|\mathcal{C}|$ has the property given in Definition 5.9. So let $n \in \mathbb{N}$ with $n \geq|\mathcal{C}|$ and $c_{1}, \ldots, c_{n} \in \mathcal{C}$. If all sums

$$
0=\sum_{\nu \in \emptyset} c_{\nu}, c_{1}, c_{1}+c_{2}, \ldots, c_{1}+\ldots+c_{n-1}
$$

are pairwise distinct, then one of the sums coincides with $c_{1}+\ldots+c_{n}$ as required. Suppose there are $k, \ell \in[0, n-1]$ with $k<\ell$ and $c_{1}+\ldots+c_{k}=c_{1}+\ldots+c_{\ell}$. Then $c_{1}+\ldots+c_{n}=c_{1}+\ldots+c_{k}+c_{\ell+1}+\ldots+c_{n}$ as required.

Lemma 5.11. Let $F$ be a monoid, $H \subset F$ a submonoid with $H^{\times}=H \cap F^{\times}$and $\mathcal{C}=\mathcal{C}^{*}(H, F)$.

If $n \in \mathbb{N}$ and $f, a_{1}, \ldots, a_{n} \in F$ with $f a_{1} \ldots a_{n} \in H$, then there exists a subset $\Omega \subset[1, n]$ such that

$$
|\Omega| \leq \mathrm{d}(\mathcal{C}) \text { and } f \prod_{\nu \in \Omega} a_{\nu} \in H
$$

Proof. By the definition of $\mathrm{d}(\mathcal{C})$, there exists a subset $\Omega \subset[1, n]$ such that $|\Omega| \leq \mathrm{d}(\mathcal{C})$ and

$$
\sum_{\nu=1}^{n}\left[a_{\nu}\right]_{H}^{F}=\sum_{\nu \in \Omega}\left[a_{\nu}\right]_{H}^{F}
$$

Since $f a_{1} \ldots a_{n} \in H$, we have

$$
f \prod_{\nu \in \Omega} a_{\nu} \in\left[f \prod_{\nu \in \Omega} a_{\nu}\right]_{H}^{F}=[f]_{H}^{F}+\sum_{\nu \in \Omega}\left[a_{\nu}\right]_{H}^{F}=[f]_{H}^{F}+\sum_{\nu=1}^{n}\left[a_{\nu}\right]_{H}^{F}=\left[f \prod_{\nu=1}^{n} a_{\nu}\right]_{H}^{F} \subset H .
$$

\section{Proposition 5.12.}

1. $\mathrm{D}(G) \leq \omega(G) \leq \mathrm{D}(G)+\mathrm{d}(\mathcal{C}(\mathcal{B}(G), \mathcal{F}(G)))$.

2. $\mathrm{D}\left(G / G^{\prime}\right) \leq \mathrm{D}(\mathcal{C}(\mathcal{B}(G), \mathcal{F}(G)))$, and equality holds if $G$ is abelian.

Proof. We set $\mathcal{C}=\mathcal{C}(\mathcal{B}(G), \mathcal{F}(G))$.

1. The first inequality follows from Lemma 5.8. For the second inequality, let $U \in \mathcal{A}(G)$, and $A_{1}, \ldots, A_{n} \in \mathcal{B}(G)$ such that

$$
U \mid A_{1} \cdot \ldots \cdot A_{n} \text { in } \mathcal{B}(G)
$$


After renumbering if necessary, we may assume that $U \mid A_{1} \cdot \ldots \cdot A_{|U|}$ in $\mathcal{F}(G)$, say $A_{1} \cdot \ldots \cdot A_{|U|}=U \cdot W$ with $W \in \mathcal{F}(G)$. Then $W \cdot A_{|U|+1} \cdot \ldots \cdot A_{n} \in \mathcal{B}(G)$. By Lemma 5.11] there is a subset $\Omega \subset[|U|+1, n]$, say $\Omega=[|U|+1, m]$, such that $|\Omega| \leq \mathrm{d}(\mathcal{C})$ and $W \cdot A_{|U|+1} \cdot \ldots \cdot A_{m} \in \mathcal{B}(G)$. Thus we obtain

$$
A_{1} \cdot \ldots \cdot A_{m}=U \cdot\left(W \cdot A_{|U|+1} \cdot \ldots \cdot A_{m}\right)
$$

and $m \leq|U|+\mathrm{d}(\mathcal{C}) \leq \mathrm{D}(G)+\mathrm{d}(\mathcal{C})$.

2. By Theorem 3.8 $1, G / G^{\prime}$ is an isomorphic to a subsemigroup of $\mathcal{C}$. Clearly, this implies that $\mathrm{D}\left(G / G^{\prime}\right) \leq \mathrm{D}(\mathcal{C})$. Suppose that $G$ is abelian. Then $\left|G^{\prime}\right|=1$ and $G / G^{\prime}$ is isomorphic to $G$. Furthermore, $\mathcal{B}(G) \hookrightarrow \mathcal{F}(G)$ is a divisor theory, the class semigroup $\mathcal{C}$ is isomorphic to the class group of $\mathcal{B}(G)$ by Lemma 2.1, and the class group of $\mathcal{B}(G)$ is isomorphic to $G$. Thus equality holds.

\section{REFERENCES}

[1] S.D. Adhikari, W. Gao, and Guoqing Wang, Erdös-Ginzburg-Ziv theorem for finite commutative semigroups, Semigroup Forum 88 (2014), 555 - 568.

[2] N.R. Baeth and D. Smertnig, Factorization theory: From commutative to noncommutative settings, J. Algebra 441 (2015), $475-551$

[3] J. Bass, Improving the Erdös-Ginzburg-Ziv theorem for some non-abelian groups, J. Number Theory 126 (2007), 217 236.

[4] F.E. Brochero Martínez and S. Ribas, Extremal product-one free sequences in Dihedral and Dicyclic Groups, Discrete Mathematics(2017), https://doi.org/10.1016/j.disc.2017.09.024.

[5] F. Chen and S. Savchev, Long minimal zero-sum sequences in the groups $C_{2}^{r-1} \oplus C_{2 k}$, Integers 14 (2014), Paper A23.

[6] K. Cziszter and M. Domokos, On the generalized Davenport constant and the Noether number, Central European J. Math. 11 (2013), $1605-1615$.

[7] _ Groups with large Noether bound, Ann. Inst. Fourier (Grenoble) 64 (2014), 909 - 944.

[8] The Noether number for the groups with a cyclic subgroup of index two, J. Algebra 399 (2014), 546 - 560.

[9] K. Cziszter, M. Domokos, and A. Geroldinger, The interplay of invariant theory with multiplicative ideal theory and with arithmetic combinatorics, Multiplicative Ideal Theory and Factorization Theory, Springer, 2016, pp. 43 - 95.

[10] K. Cziszter, M. Domokos, and I. Szöllősi, The Noether number and the Davenport constants of the groups of order less than 32, arXiv:1702.02997.

[11] Y. Fan and Q. Zhong, Products of $k$ atoms in Krull monoids, Monatsh. Math. 181 (2016), $779-795$.

[12] W. Gao and A. Geroldinger, Zero-sum problems in finite abelian groups: a survey, Expo. Math. 24 (2006), 337 - 369.

[13] W. Gao and Yuanlin Li, The Erdös-Ginzburg-Ziv theorem for finite solvable groups, J. Pure Appl. Algebra 214 (2010), $898-909$.

[14] W. Gao and Zaiping Lu, The Erdös-Ginzburg-Ziv theorem for dihedral groups, J. Pure Appl. Algebra 212 (2008), 311 -319 .

[15] A. Geroldinger, Additive group theory and non-unique factorizations, Combinatorial Number Theory and Additive Group Theory (A. Geroldinger and I. Ruzsa, eds.), Advanced Courses in Mathematics CRM Barcelona, Birkhäuser, 2009 , pp. $1-86$.

[16] - Sets of lengths, Amer. Math. Monthly 123 (2016), 960 - 988.

[17] A. Geroldinger and D.J. Grynkiewicz, The large Davenport constant I: Groups with a cyclic index 2 subgroup, J. Pure Appl. Algebra 217 (2013), $863-885$.

[18] A. Geroldinger and F. Halter-Koch, Non-Unique Factorizations. Algebraic, Combinatorial and Analytic Theory, Pure and Applied Mathematics, vol. 278, Chapman \& Hall/CRC, 2006.

[19] A. Geroldinger and W. Hassler, Arithmetic of Mori domains and monoids, J. Algebra 319 (2008), 3419 - 3463.

[20] A. Geroldinger and F. Kainrath, On the arithmetic of tame monoids with applications to Krull monoids and Mori domains, J. Pure Appl. Algebra 214 (2010), 2199 - 2218.

[21] A. Geroldinger, S. Ramacher, and A. Reinhart, On v-Marot Mori rings and C-rings, J. Korean Math. Soc. 52 (2015), $1-21$.

[22] A. Geroldinger and W. A. Schmid, A realization theorem for sets of distances, J. Algebra, 481 (2017), $188-198$.

[23] A. Geroldinger and Q. Zhong, The catenary degree of Krull monoids II, J. Australian Math. Soc. 98 (2015), 324 - 354.

[24] D.J. Grynkiewicz, The large Davenport constant II: General upper bounds, J. Pure Appl. Algebra 217 (2013), 2221 2246.

[25] _ Structural Additive Theory, Developments in Mathematics, vol. 30, Springer, 2013.

[26] Dongchun Han, The Erdös-Ginzburg-Ziv Theorem for finite nilpotent groups, Archiv Math. 104 (2015), 325 - 332.

[27] Dongchun Han and Hanbin Zhang, Erdös-Ginzburg-Ziv Theorem and Noether number for $C_{m} \ltimes_{\varphi} C_{m n}$, arXiv:1707.03639.

[28] F. Kainrath, Arithmetic of Mori domains and monoids: The Global Case, Multiplicative Ideal Theory and Factorization Theory, Springer, 2016, pp. $183-218$. 
[29] J.E. Olson and E.T. White, Sums from a sequence of group elements, Number Theory and Algebra (H. Zassenhaus, ed.), Academic Press, 1977, pp. 215 - 222.

[30] A. Reinhart, On integral domains that are C-monoids, Houston J. Math. 39 (2013), 1095 - 1116.

[31] W.A. Schmid, The inverse problem associated to the Davenport constant for $C_{2} \oplus C_{2} \oplus C_{2 n}$, and applications to the arithmetical characterization of class groups, Electron. J. Comb. 18(1) (2011), Research Paper 33.

[32] _ Some recent results and open problems on sets of lengths of Krull monoids with finite class group, Multiplicative Ideal Theory and Factorization Theory (S.T. Chapman, M. Fontana, A. Geroldinger, and B. Olberding, eds.), Springer, 2016 , pp. $323-352$.

[33] D. Smertnig, Sets of lengths in maximal orders in central simple algebras, J. Algebra 390 (2013), $1-43$.

[34] Guoquing Wang, Davenport constants for semigroups II, J. Number Theory 153 (2015), 124 - 134.

[35] Guoquing Wang and Weidong Gao, Davenport constants for semigroups, Semigroup Forum 76 (2008), $234-238$.

Institute for Mathematics and Scientific Computing, University of Graz, NAWi Graz, Heinrichstrasse 36 , 8010 Graz, Austria

E-mail address: junseok.oh@uni-graz.at 